Pacific Journal of Mathematics

A CHARACTERIZATION OF THE UNITARY AN CHARACTERISTIC AT LEA 


\section{A CHARACTERIZATION OF THE UNITARY AND SYMPLECTIC GROUPS OVER FINITE FIELDS OF CHARACTERISTIC AT LEAST 5}

\section{MichaEL ASChBACHER}

The following characterization is obtained:

Theorem. Let $G$ be a finite group generated by a conjugacy class $D$ of subgroups of prime order $p \geqq 5$, such that for any choice of distinct $A$ and $B$ in $D$, the subgroup generated by $A$ and $B$ is isomorphic to $Z_{p} \times Z_{p}, L_{2}\left(p^{m}\right)$ or $S L_{2}\left(p^{m}\right)$, where $m$ depends on $A$ and $B$. Assume $G$ has no nontrivial solvable normal subgroup. Then $G$ is isomorphic to $S p_{n}(q)$ or $U_{n}(q)$ for some power $q$ of $p$.

A much larger class of groups satisfies the analogous property for $p=2$ or 3 , including many of the sporatic simple groups. The classification for $p=2$ appears in [3]. The classification for $p=3$ is incomplete, but a partial solution appears in [4].

For the most part the proof here mimics that in the papers mentioned above. The exception comes in handling certain degenerate cases. This is accomplished in $\S 4$ by first showing a minimal counter example possesses a doubly transitive permutation representation, and then utilizing numerous results on doubly transitive groups.

1. Notation. In general $G$ is a finite group and $D$ a $G$ invariant collection of subgroups generating $G$. $G$ acts on $D$ by conjugation with this representation denoted by $G^{D}$. If $\alpha \cong D$ is a set of imprimitivity for this action we define

$$
\begin{aligned}
D_{\alpha} & =\left\{\beta \in \alpha^{G}:[\alpha, \beta]=1, \alpha \neq \beta\right\} \\
\alpha^{\perp} & =\{\alpha\} \cup D_{\alpha} \\
A_{\alpha} & =\alpha^{G}-\alpha^{\perp} \\
V_{\alpha} & =\left\{\beta \in \alpha^{G}: \alpha^{\perp}=\beta^{\perp}\right\} \\
W_{\alpha} & =\left\{\beta \in \alpha^{G}: D_{\alpha}=D_{\beta}\right\} \\
D_{\alpha}^{*} & =\left\{B: B \in \beta \in D_{\alpha}\right\} .
\end{aligned}
$$

For $\Omega \subseteq \alpha^{G}, \mathscr{D}(\Omega)$ is the graph with point set $\Omega$ and edges $\left(\alpha^{g}\right.$, $\left.\alpha^{h}\right)$ where $\alpha^{g} \in D_{\alpha^{h}} . \mathscr{B}(\Omega)$ is the geometry with point set $\Omega$ and block set $\left\{\beta^{\perp} \cap \Omega: \beta \in \Omega\right\}$. For $\alpha, \beta \in \Omega$ the line through $\alpha$ and $\beta$ in $\mathscr{B}(\Omega)$ is

$$
\alpha * \beta=\bigcap_{\gamma \in \alpha \perp \cap \beta \perp \cap \Omega}\left(\gamma^{\perp} \cap \Omega\right)
$$

$\alpha * \beta$ is singular if $\beta \in D_{\alpha}$ and hyperbolic otherwise. 
A triangle is a triple $(A, B, C)$ with $A \in D, C \in D_{A}$, and $B \in A_{A} \cap A_{C}$.

If $G$ is a permutation group on a set $\Omega, \Delta \subseteq \Omega$ and $X \subseteq G$, then $X_{\Delta}, X(\Delta)$ is the pointwise, global stabilizer of $\Delta$ in $X$ respectively. $X^{\lrcorner}=X(\Delta) / X_{\Delta}$ with induced permutation representation. $F(X)$ is the set of fixed points of $X$.

$O_{\infty}(G)$ is the largest normal solvable subgroup of $G$.

All groups are finite.

2. Locally $D$-simple groups. Let $G$ be a finite group and $D$ a collection of subgroups of $G$ such that $D^{G}=D$. Represent $G$ as a permutation group on $G$ by conjugation. $G$ is said to be $D$-simple if $G$ is generated by any $G$ invariant subset of $D . \quad G$ is locally $D$-simple if $D$ generates $G$ and for any $A$ and $B$ in $D$ either $[A, B]=1$ or $\langle A, B\rangle$ is generated by $A^{\langle A, B\rangle}$. $\alpha$ is a set of imprimitivity for $G^{D}$ if $\alpha \cap \alpha^{\prime}=\varnothing$ for $g \in G-N_{G}(\alpha)$, and $\varnothing \neq \alpha=\langle\alpha\rangle \cap D \neq D$.

Lemma 2.1. Let $G$ be locally D-simple and $\Delta$ a $G$ invariant subset of $D$. Then

(1) If $H$ is a D-subgroup of $G$ then $H$ is locally $(H \cap D)$-simple.

(2) If $\alpha$ is a homomorphism of $G$ then $G \alpha$ is locally $D \alpha$-simple.

(3) Let $\Gamma=\langle\Delta\rangle \cap D$. Then $[\Gamma, D-\Gamma]=1$.

(4) If $G^{A}$ is transitive then $\langle\Delta\rangle^{A}$ is transitive.

(5) If $D \cap Z(G)$ is empty and $G=\langle\Delta\rangle$ for some orbit $\Delta$ of $G^{\prime \prime}$, then $G$ is D-simple.

Proof. (1) and (2) are straightforward. Let $H=\langle\Delta\rangle$. Then $H \unlhd$ $G$. Let $A \in \Gamma, B \in D-\Gamma$ and assume $[A, B] \neq 1$. Let $X=\langle A, B\rangle$. Then $X=\left\langle A^{X}\right\rangle \leqq H$ so $B \in \Gamma$, contradicting the choice of $B$. Therefore, (3) holds.

Assume $G^{s}$ is transitive. Let $K=\langle D-\Gamma\rangle$. Then by (3) $G$ is the central product of $H$ and $K$ so for $A \in \Delta, \Delta=A^{G}=A^{K I I}=A^{H I}$. Thus (4) holds.

Finally assume $G^{\Delta}$ is transitive, $G=\langle\Delta\rangle$ and $Z(G) \cap D$ is empty. Suppose $\Omega$ is an orbit of $G^{D}$ with $K=\langle\Omega\rangle \neq G$. Then as $G=\langle\Delta\rangle, \Delta \cap K$ is empty, so by (3), $[\Delta, \Omega]=1$. Thus $\Omega$ is centralized by $G$, a contradiction. Thus (5) holds.

Lemma 2.2. Let $G$ be locally D-simple and $\alpha$ a set of imprimitivity for $G^{D}$. Then

(1) If $A \in \alpha, B \in \alpha^{g} \neq \alpha$ and $[A, B]=1$, then $\left.] \alpha, \alpha^{g}\right]=1$.

(2) $\left\langle\alpha^{G}\right\rangle$ is locally $\langle\alpha\rangle^{G}$-simple.

Proof. (1) $A=A^{B} \in \alpha^{B}$, so $\alpha^{B}=\alpha$. Thus 2.1.3 applied to $\langle\alpha, B\rangle$ implies $[\alpha, B]=1$. But now the same argument shows $\left[\alpha^{g}, C\right]=1$ for each $C$ in $\alpha$. (2) Let $H=\langle\alpha\rangle \neq K=\left\langle\alpha^{g}\right\rangle$, and $X=\langle H, K\rangle$. Assume 
$[H, K] \neq 1$ and let $A \in \alpha, B \in \alpha^{g}$. Then by (1), $[A, B] \neq 1$ so $B \in\left\langle A^{\langle A, B\rangle}\right\rangle \leqq$ $\left\langle H^{X}\right\rangle$. Thus $X=\left\langle H^{x}\right\rangle$.

Lemma 2.3. Let $G$ be locally $D$-simple with $G^{D}$ transitive, and A abelian. Then

(1) Either $V_{A}$ or $W_{A}$ equals $\{A\}$.

(2) $V_{A}$ and $W_{A}$ are sets of imprimitivity for $G^{D}$.

(3) $V_{V_{A}}=\left\{V_{A}\right\}$ and $W_{W_{A}}=\left\{W_{A}\right\}$.

Proof. Straightforward.

Lemma 2.4. Let $G$ be locally $D$-simple with $G^{D}$ transitive and $\mathscr{D}(D)$ connected. Let $A \in D$. Then $A$ is contained in a unique maximal set of imprimitivity $\alpha$ of $G$ and $\left\langle D_{\alpha}^{*}\right\rangle$ is $D_{\alpha}^{*}$-simple.

Proof. Let $H=\left\langle D_{A}\right\rangle, \pi$ an orbit of $H$ of maximal length on $D_{A}$, $\Delta=(\langle\pi\rangle-Z(\langle\pi\rangle)) \cap D, \Gamma=N_{D}(\Delta)$ and $\alpha=\langle\Gamma-\Delta\rangle \cap D$. As $\mathscr{D}(D)$ is connected, $|\pi|>1$, so $\Delta$ is nonempty. We will show $\alpha$ has the properties claimed in the conclusion of the lemma.

By 2.1.3, $[\alpha, \Delta]=1$. By 2.1.4 $\langle\pi\rangle$ is transitive on $\pi$. Thus transitivity of $G^{D}$ and maximality of $|\pi|$ imply $\pi$ is an orbit of $\left\langle D_{B}\right\rangle$ on $D_{B}$, for $B \in \alpha$. Therefore $B^{\perp} \subseteq \Gamma$.

Suppose $B \in \alpha \cap \alpha^{g} \neq \alpha$. Then $\Delta \subseteq B^{\perp} \subseteq \Gamma^{g}=\alpha^{g} \cup \Delta^{g}$. Now $\langle\pi\rangle$ is transitive on $\pi$ so either $\pi \cong \Delta^{g}$ or $\pi \cong \alpha^{g}$. If $\pi \cong \Delta^{g}$ then $\Delta \leqq$ $\langle\pi\rangle \subseteq\left\langle\Delta^{g}\right\rangle$, so $\Delta=\Delta^{g}$ and therefore $\alpha=\alpha^{g}$, a contradiction. Thus $\pi \sqsubseteq \alpha^{g}$, so $\Delta \leqq\langle\pi\rangle \sqsubseteq\left\langle\alpha^{g}\right\rangle$ and therefore $\Delta \leqq \alpha^{g}$.

So $\Gamma \leqq \alpha \cup \alpha^{g}$. Further $\Delta^{g} \leqq \alpha$, so $\alpha^{g} \leqq C^{\perp} \subseteq \Gamma$ for $C \in \Delta^{g}$. Thus $\Gamma=$ $\alpha \cup \alpha^{g}$. From the last remark of the second paragraph it follows that $\Gamma$ is a component of $\mathscr{D}(D)$, contradicting the hypothesis that $\mathscr{D}(D)$ is connected.

It follows that $\alpha$ is a set of imprimitivity for $G^{D}$. By 2.2.1, $D_{\alpha}^{*}=$ $D_{A}-\alpha=\Delta-\alpha$. By construction, $Z(\langle\Delta\rangle) \cap \Delta$ is empty, so $D_{\alpha}^{*}=\Delta$ and by $2.1 .5,\langle\Delta\rangle$ is $\Delta$-simple.

Finally let $\beta$ be a set of imprimitivity for $G$ containing $A$. $\Delta$ centralizes $A$, so $\Delta$ normalizes $\beta$. If $B \in \beta \cap \Delta$ then as $K=\langle\Delta\rangle$ is $\Delta$ simple, $\Delta \subseteq\left\langle B^{K}\right\rangle \leqq\left\langle\beta^{K}\right\rangle=\langle\beta\rangle$. Thus $\Delta \leqq \beta$. As $N_{G}(\beta)$ is transitive on $\beta, \alpha \subseteq D_{\alpha^{g}} \leqq \beta$ for $\alpha^{g} \in D_{\alpha}$. Thus $A^{\perp} \subseteq \beta$, and transitivity of $N_{G}(\beta)^{\beta}$ implies $\beta$ is a component of $\mathscr{D}(D)$, contradicting the hypothesis that $\mathscr{D}(D)$ is connected.

So $\beta \cap \Delta$ is empty and by 2.1.3, $[\beta, \Delta]=1$. Thus $\beta \cong N_{D}(\Delta)-$ $\Delta=\alpha$. Thus $\alpha$ is maximal as claimed.

Lemmas 2.6 and 2.7 are from $\S 2$ of [4]. 2.6 is a slight generalization of its counterpart, but the same proof goes through. 
Lemma 2.6. Let $G$ be locally $\Omega$-simple, let $\Lambda \subseteq \Omega$, and let $H$ be a $\Omega$-subgroup of $G$. Assume

(i) $H$ takes the edge set of $\mathscr{D}(\Lambda)$ onto the edge set of $\mathscr{D}(\Omega)$ under conjugation.

(ii) There exists a partition $\Lambda=\Sigma \Lambda_{i}$ of $\Lambda$ such that if $\alpha^{h} \in \Lambda$ for some $\alpha \in \Lambda_{i}, h \in H$, then there exists $r \in N_{H}\left(\Lambda_{i}\right)$ with $\alpha^{h}=\alpha^{r}$.

Let $\bar{G}$ be a second group satisfying the hypothesis of $G$ for which there exists a permutation isomorphism $T$ of $H^{s} \bar{H}^{\bar{a}}$ and an isomorphism $S$ of $\mathscr{D}(\Lambda)$ and $\mathscr{D}(\bar{\Lambda})$ such that

(iii) $T$ restricted to $N_{H}\left(\Lambda_{i}\right)$ commutes with $S$ and $N_{H}(\alpha) T=N_{\bar{H}}(\alpha S)$ for each $\alpha \in \Lambda$.

Then $S$ extends to an isomorphism of $\mathscr{D}(D)$ and $\mathscr{D}(\bar{D})$.

A triangle in $D$ is a triple $(A, B, C)$ with $A \in D, C \in D_{A}$, and $B \in$ $A_{A} \cap A_{C} . \quad D$ is locally conjugate in $G$ if for $A, B \in D, A$ is conjugate to $B$ in $\langle A, B\rangle$, or $[A, B]=\perp$.

Lemma 2.7. Let $\Omega$ be locally conjugate in $G$ with $G^{\Omega}$ primitive and $\mathscr{D}(\Omega)$ connected. Assume

$\left(^{*}\right)$ If $(\alpha, \beta, \gamma)$ is a triangle and $X=\langle\alpha, \beta, \gamma\rangle$, then $\beta^{\perp} \cap X \subseteq$ $\beta^{\langle\alpha \perp \cap X\rangle}$ and $\beta^{\gamma} \subseteq\left(\beta^{\perp} \cap X\right)^{\alpha}$.

Then $\left\langle\alpha^{\perp}\right\rangle$ is transitive on $A_{\alpha}$ and $G^{\Omega}$ is rank 3 .

3. $p$-transvections. Let $G$ be a finite group, $p$ a prime. A set of $p$-transvections of $G$ is a $G$ invariant collection $D$ of subgroups generating $G$ such that for any $A, B \in D,|A|=p$ and $\langle A, B\rangle$ is the homomorphic image of a subgroup of $S L_{2}\left(p^{n}\right)$, with $n$ and the image depending on $A$ and $B$.

If $p=2$ then $D$ is a set of odd transpositions. Groups generated by odd transpositions have been classified [3]; they include the sporatic simple groups discovered by Fischer plus many infinite classes of simple groups. Conway's sporatic simple group $\cdot 1$ is generated by 3 -transvections, as is the Hall-Janko group and Suzuki's sporatic simple group.

LEMma 3.1. Let $D$ be a set of p-transvections of $G, p>2$, and let $M=O_{\infty}(G)$. Then

(1) $G$ is locally $D$-simple

(2) If $G$ is a p-group then $G$ is abelian

(3) If $G=M$ is not a p-group then $p=3$ and $G$ is a $\{2,3\}$ group

(4) If $p>3$ then $M / O_{p}(G)=Z\left(G / O_{p}(G)\right)$.

(5) Let $M=1$. Then $G$ is a simple unless $p=3$ and $G \cong$ $P G U_{3 n}(2)$.

Proof. Let $A, B \in D,[A, B] \neq 1$. Set $X=\langle A, B\rangle$. Then $X$ is isomorphic to $S L_{2}\left(p^{n}\right)$ or $L_{2}\left(p^{n}\right)$ unless $p=3$ and $X \cong S L_{2}(5)$ or $L_{2}(5)$. 
This implies (1) and (2). If $G=M$ then as $L_{2}(q)$ is simple for $q>3$, $X$ must be isomorphic to $S L_{2}(3)$ or $A_{4}$. Therefore, 4.1 of [4] yields (3).

Assume $p>3$. To prove (4) we may assume $O_{p}(G)=1$. Let $Q$ be a minimal normal subgroup of $G$ contained in $M$. Then $Q$ is a $q$-subgroup for some prime $q \neq p$. If $A$ centralizes $Q$ then $Q$ is in the center of $G=\langle D\rangle$, so we can assume $[A, Q] \neq 1$. But then $\left\langle A^{Q}\right\rangle \leqq A Q$ is a solvable $D$-subgroup whose order is divisible by $q$, contradicting (3).

Finally assume $M=1$ and let $H$ be a minimal normal subgroup of $G$. If $A \not \leq H$ and $x \in H$ then $\left\langle A, A^{x}\right\rangle$ has a normal subgroup of index $p$, so either $A^{x} \in A^{\perp}$ or $\left\langle A, A^{x}\right\rangle \cong S L_{2}(3)$ or $A_{4}$. If $A^{H} \cong A^{\perp}$ then $[H, A]$ is a normal abelian subgroup of $H$, so $[H, A]=1$. Thus $H$ is centralized by $G=\langle D\rangle$, a contradiction. Therefore, if $A \not \subset H$, then [4] implies $A H \cong P G U_{3 n}(2)$. $P G U_{m}(2)$ is normal in Aut $U_{m}(2)$ so $G=C_{G}(H) H A$. By induction on $|G|, G / H \cong C_{G}(H) A \cong Z_{p}$ or $P G U_{3 m}(2)$. But now [4] implies the latter case does not occur.

So we can take $A \leqq H$. So $G=\langle D\rangle=H$ is simple.

The proof of the following lemma is due to David Wales.

LEMmA 3.2. Let $G \cong L_{2}(q)$ or $S L_{2}(q), q=p^{m}$ odd, with Sylow $p$ subgroup $P$. Assume $G$ acts irreducibly on a n-dimensional vector space over $G F(p)$, such that $n=2 \operatorname{dim} C_{V}(P)$ and $P$ acts semiregularly on $V-C_{V}(P)$. Then $G \cong S L_{2}(q), n=2 m$, and $G$ acts in its natural representation on $V$.

Proof. Let $B$ be a basis of $V$, and $G F(r)$ the splitting field for the representation of $G$ on $V$. Extend the action of $G$ to a vector space $W$ over $G F(r)$ with basis $B$. $W$ is the sum of $k$ absolutely irreducible $G$-invariant subspaces $W_{i}$ of $W$. By inspection of the irreducible representations of $S L_{2}(q)$ (e.g. $\left.\S 30,[7]\right), \operatorname{dim} C_{W_{i}}(P)=1$ for all $i$. Thus as $n=2 \operatorname{dim} C_{V}(P)$ and $P$ acts semiregularly on $V-C_{V}(P), \operatorname{dim} C_{W_{i}}(P)=$ 2. Again by inspection of the representations of $S L_{2}(q), q=r, G \cong$ $S L_{2}(q)$, and $G$ acts in its natural fashion on $W_{1}$. Further $G^{W_{i}}, 1 \leqq$ $i \leqq k$, are the $m$ equivalent representations obtained from $G^{W_{i}}$ by Aut $G F(q)$. Thus $n=2 m$ and $G$ acts in its natural fashion on $V$.

LEMma 3.3. Let $D$ be a class of p-transvections of $G, p$ odd, with $G / O_{\infty}(G) \cong L_{2}(q)$. Let $M=O_{p}(G), A \in D, m=\left|A^{M}\right|$ and $Z=Z(G)$. Assume $O_{\infty}(G) / M=Z(G / M)$. Then for some $B \in D, G=M X$ where $X=$ $\langle A, B\rangle \cong S L_{2}(q), Z=\left[A^{\perp}, M\right] \cap\left[B^{\perp}, M\right], M=[A, M][B, M],|M / Z|=m^{2}$ where $m=\left|A^{M}\right|, Z=C_{M}(x)$ for any $p^{\prime}$-element of $X$, and $[M, \beta]$ is transitive on $A^{M}$.

Proof. As $G / O_{\infty}(G) \cong L_{2}(q)$ there exists $B \in D$ with $X=\langle A, B\rangle \cong$ $L_{2}(q)$ or $S L_{2}(q)$. Let $\alpha=A^{\perp} \cap X$, and $\Omega=\alpha^{X}$. Let $K=\Pi_{\Omega}[M, \beta]$. 
By 3.1, $[M, \alpha]$ is elementary abelian, $G=\langle[M, \alpha], X\rangle$ normalizes $K$ and $[A, M / K]=1$. So $M=K$. As $X^{2}$ is doubly transitive, $Z_{0}=$ $[M, \alpha] \cap[M, \beta]=[M, \gamma] \cap[M, \delta]$ for all pairs $(\alpha, \beta),(\lambda, \delta)$ from $\Omega$. So as $[M, \alpha]$ is abelian, $Z_{0} \leqq Z$. Thus we can assume $Z_{0}=1$. Therefore, $M$ is elementary abelian. $A$ is in $m$ groups $\langle A, C\rangle, C \in B^{I I}$, so there are $m^{2}$ total $D$-subgroups isomorphic to $L_{2}(q)$ or $S L_{2}(q)$. Set $\bar{G}=G / Z$. $Z=C_{M I}(X)$, so $m^{2} \geqq\left|\bar{X}^{\bar{G}}\right|=|\bar{M}| \geqq|[\bar{M}, \alpha][\bar{M}, \beta]|$. On the other hand $m=\left|A^{\bar{M}}\right| \leqq|[\bar{M}, \alpha]|$, so $m=|[\bar{M}, \alpha]|, \bar{M}=[\bar{M}, \alpha][\bar{M}, \beta]$, and $A^{\bar{M}}=A^{[\bar{M}, \beta]}$. Lemma 3.2 implies $\bar{X} \cong S L_{2}(q)$ and $C_{\bar{H}}(x)=1$ for all $p^{\prime}$-elements $x \in X$. So it suffices to show $Z=1$. Let $\langle u\rangle=Z(X)$. Then $M=Z[M, u]$, so $D \subseteq X[M, u] \unlhd G$. Thus $Z=1$.

Lemma 3.4. Let $D$ be a class of p-transvections of $G, p$ odd, with $M=O_{p}(G), X$ a $D$-subgroup with $X \mid Z(X) \cong U_{3}(q)$, and $G=M X$. Let $Z=Z(G), A \in M$ and $m=|A M|$. Then $Z \leqq\left[A^{\perp}, M\right]$ and $|M| Z \mid=m^{3}$.

Proof. Let $X=\left\langle A_{i}, 1 \leqq i \leqq 3\right\rangle, A=A_{1}$, let $\alpha_{i}=A_{i}^{\llcorner} \cap X$ and $\Omega=$ $\alpha^{X}$. Set $Z_{0}=[\alpha, M] \cap\left[\alpha_{2}, M\right]$. As $X^{2}$ is doubly transitive $Z_{0}=[\beta$, $M] \cap[\gamma, M]$ for $\beta, \gamma \in \Omega$. $[\alpha, M]$ is abelian so $G=\left\langle X, A^{H}\right\rangle$ centralizes $Z_{0}$. Thus we can assume $Z_{0}=1$.

Set $N=\prod_{i=1}^{3}\left[M, \alpha_{i}\right]$. By 3.3, $\left[M, \alpha_{i}\right]^{\alpha_{j}} \leqq\left[M \alpha_{i}\right]\left[M, \alpha_{j}\right]$, so $N$ is normalized by $G=\left\langle\alpha_{1}, \alpha_{2}, \alpha_{3}, M\right\rangle$. $A$ centralizes $M / N$, so $M=N$. As $Z_{0}=1, M$ is abelian. Let $u$ be the involution in $\left\langle\alpha_{1}, \alpha_{2}\right\rangle$ and $v$ the involution in $\left\langle\alpha_{2}, \alpha_{3}\right\rangle$. We may assume $[u, v]=1$. $M=C_{M}(u) \times$ $[M, u]$ and by 3.3, $C_{M}(u)=C_{M}\left(\alpha_{1}\right) \cap C_{M}\left(\alpha_{2}\right)$ and $[M, u]=\left[M, \alpha_{1}\right]\left[M, \alpha_{2}\right]$. Therefore, $C_{M}(u) \cap C_{M I}(v)=Z$ and as $X$ has one class of involutions, $\left|C_{M I}(u) / Z\right|^{3}=|M / Z|=\left|C_{M}(u) / Z\right| m^{2}$. So $|M / Z|=m^{3}$, and as $|M| \leqq m^{3}$, $Z=1$. That is $Z=Z_{0} \leqq[A, M]$.

4. Groups with $\mathscr{D}(D)$ disconnected. This section consists of a proof of the following theorem:

THEOREM 4.1. Let $D$ be a conjugacy class of $p$-transvections, $p \geqq$ 5, of the group $G$. Assume $\mathscr{D}(D)$ is disconnected and $O_{\infty}(G)=1$. Then $G \cong L_{2}(q)$ or $U_{3}(q)$ for some power $q$ of $p$.

Throughout $\S 4, G$ is a counterexample of minimal order to Theorem 4.1. For $A \in D$ let $\bar{A}$ be the component of $\mathscr{D}(D)$ containing $A$. Let $\bar{D}$ be the set of components. Write $A \sim B$ if $A, B \in D$ and $\langle A, B\rangle$ is isomorphic to $L_{2}(p)$ or $S L_{2}(p)$. For $\bar{A} \neq \bar{B}$ define

$$
\Gamma_{A \bar{B}}=\{C \in \bar{A}: A \sim E \sim C \text { for some } E \in \bar{B}\} .
$$

Now for $\bar{A} \neq \bar{B}, A \sim B$ if and only if $\bar{A} \cup \bar{B}^{A}=\bar{B} \cup \bar{A}^{B}$. Thus if $A \sim$ 
$B$ then $X=\left\langle\Gamma_{A \bar{B}}, \Gamma_{B \bar{A}}\right\rangle$ acts on $\Gamma=\bar{A} \cup \bar{B}^{A}$ of order $p+1$, so $Y=$ $\left\langle\Gamma_{A \vec{B}}\right\rangle=A Y_{\Gamma}$ and $X=\langle Y, B\rangle=\langle A, B\rangle X_{\Gamma}$. By 3.1, $X_{\Gamma}=0_{\infty}(X)$ and $Y$ is a $p$-group. Further for fixed $\bar{B} \neq \bar{A}$, the sets $\Gamma_{C \bar{B}}, C \in \bar{A}$, partition $\bar{A}$.

Let $m=\left|\Gamma_{A \bar{B}}\right|$, and let $n$ be the number of classes $\Gamma_{C \bar{B}}$ in $\bar{A}$. If $m>1$ then applying 3.3 to $X$ we have that $\langle A, B\rangle$ contains a central involution $u=u(A, B)$, and $u$ centralizes only $A$ in $\Gamma_{A \bar{B}}$.

Let $C \in \bar{A} .\langle C, B\rangle$ contains $E \in \Gamma_{A \bar{B}}$ and $v=u(E, B)$ is in the center of $\langle C, B\rangle$. Indeed $v=u(C, F)$ where $C \sim F \in \bar{B} \cap\langle C, B\rangle$. As $v$ centralizes a unique member of $\Gamma_{A \bar{B}}$ and $\Gamma_{C \bar{B}}$, each member $C_{1}$ of $\Gamma_{C \bar{B}}$ determines a distinct member $E_{1}$ of $\Gamma_{A \bar{B}} \cap\left\langle C_{1}, B\right\rangle$. Thus $m=\left|\Gamma_{C \bar{B}}\right|$ for all $C \in \bar{A}$. Further $u=u\left(C_{1}, F_{1}\right)$ for some $C_{1} \in \Gamma_{C \bar{B}}, F_{1} \in \Gamma_{F \bar{A}}$. So $C_{D}(u)$ intersects each $\Gamma_{C \bar{B}}$ in $\bar{A}$ in a unique member. Set $K=\left\langle C_{D}(u)\right\rangle$ and $H=\langle K, \bar{A}\rangle$. Minimality of $G$ implies $K \cong S L_{2}(q)$ for some power $q$ of $p$. So the set $\Delta$ of components of $\mathscr{D}(D)$ containing an element of $C_{D}(u)$ has order $q+1$ and $Q=\left\langle C_{\bar{A}}(u)\right\rangle$ acts regularly on $\Delta-\{\bar{A}\}$.

Now there are $m^{2}$ involutions $u\left(A_{1}, B_{1}\right), A_{1} \in \Gamma_{A \bar{B}}, B_{1} \in \Gamma_{B \bar{A}}$, and $m^{2}$ pairs $\left(A_{1}, C_{1}\right), C_{1} \in \Gamma_{C \widetilde{B}}$, with $u\left(A_{1}, B_{1}\right)$ centralizing at most one pair. It follows there exists $u$ with $A, C \in Q$. So as $Q$ is abelian, $\langle\bar{A}\rangle$ is abelian. Notice that if $m=1$ then $A=\Gamma_{A \bar{B}} \cap\langle C, B\rangle$, so again [A, $C]=1$, and $\langle\bar{A}\rangle$ is abelian. Therefore:

LEMMA 4.2. $\langle\bar{A}\rangle$ is abelian.

Let $\langle c\rangle=C \in \bar{A}$. We have shown there is an $\langle e\rangle=E \in C_{\bar{A}}(u) \cap \Gamma_{C \bar{B}}$, and we can choose $e$ such that $\bar{B}^{c}=\bar{B}^{e}$. Thus as $\langle\bar{A}\rangle$ is abelian, $\bar{B}^{2 c}=$ $\bar{B}^{c Q}=\bar{B}^{e Q}=\bar{B}^{2}$, so $H$ acts on $\Delta=\bar{A} \cup \bar{B}^{2}$, and $H=K H_{\Delta}=K O_{p}(H)$ by 3.1 .

Summarizing:

Lemma 4.3. (1) If $m>1$ then $\langle A, B\rangle$ contains a central involution $u$. (2) If $\langle A, B\rangle$ contains a central involution $u$ then $\langle\bar{A}, \bar{B}\rangle=$ $H=\left\langle C_{D}(u)\right\rangle 0_{p}(H)$ with $\left\langle C_{D}(u)\right\rangle \cong S L_{2}(q)$ for some power $q$ of $p$.

Let $J=N_{G}(\bar{A}), I=C_{G}(\bar{A})$. For $X \subseteq G$ let $F(X)$ be the set of points in $\bar{D}$ fixed by $X$.

Lemma 4.4. Assume $u$ is an involution in the center of $\langle A, B\rangle$. Then

(i) If $v$ is an involution in the center of $\langle A, C\rangle$ with $[u, v]=1$, then $u=v$.

(ii) $J=O(J) C_{J}(u)$.

Proof. Set $H=\left\langle C_{D}(u)\right\rangle$. Let $v$ be as in (i). Then $v$ acts on $H$ and fixes $\bar{A}$. There are $q+1$ members of $\bar{D}$ intersecting $H$, and $q+1$ is even, by 4.3. Thus $v$ fixes a second member $\bar{E} \neq \bar{A}$ of $\bar{D}$ 
with $\bar{E} \cap H \neq \varnothing$. As $H \cong S L_{2}(q), v$ centralizes an element $E$ of $\bar{E}$. Thus $\langle u\rangle=Z(\langle A, E\rangle)=\langle v\rangle$, yielding (i). (i) and Glauberman's $Z^{*}$ theorem imply (ii).

Lemma 4.5. Assume $m(\bar{A}, \bar{B})=1$ with $A \sim B$. Let $x \in\langle A, B\rangle$ fix $\bar{A}$ and $\bar{B}$. Then

(1) $B=\bar{B}(A)$ is the unique element of $\bar{B}$ with $A \sim B$.

(2) $x$ acts as scalar multiplication in $G F(p)$ on $Q=\langle\bar{A}\rangle$.

(3) Assume $y \in J$ has scalar action on $Q$ and fixes $\bar{B}$. Then $y$ has the same action on $\langle\bar{B}\rangle$ and if $|x I / I|>2$ then $F(x)=\{\bar{A}, \bar{B}\}$.

(4) If $\langle A, C\rangle \cong L_{2}\left(p^{n}\right)$ or $S L_{2}\left(p^{n}\right), n$ odd, for all $C \in \bar{B}$, then $\langle\bar{A}$, $\bar{B}\rangle \cong L_{2}(q)$ or $S L_{2}(q)$.

(5) If $p=5$ and $\langle A, C\rangle \cong L_{2}\left(p^{n}\right)$ or $S L_{2}\left(p^{n}\right)$, $n$ even, for some $C \in \bar{B}$ then there exists $y$ with $|I y / I|=4$ inducing scalar action on $Q$ and $\langle\bar{B}\rangle$.

(6) $m(\bar{A}, \bar{C})=1$ for all $\bar{C} \neq \bar{A}$.

Proof. (1) is just a restatement of $m(\bar{A}, \bar{B})=1$. Let $C \in \bar{A} . \quad\langle C$, $B\rangle$ contains an element $A_{1}$ of $D$ centralizing $C$ with $A_{1} \sim B$. Thus by (1), $A_{1}=\bar{A}(B)=A$. So $x \in\langle A, B\rangle \leqq\langle C, B\rangle$ and thus has the same action on $C$ as on $A$. This yields (2). Notice that (2) implies $J=I C_{J}(x)$.

Assume $y \in J$ is as in the hypothesis of (3). Then for $C \in \bar{A}, y$ fixes $C$ and therefore $\bar{B}(C)$. So $y$ acts on $\langle C, B\rangle$ with scalar action on $\bar{B} \cap\langle C, B\rangle$. So $y$ acts on $\bar{B}$ as on $\bar{A}$.

Assume $y$ has order $r^{n}$ for some prime $r, r$ dividing $p-1$, and $\bar{C} \in F(y)-\{\bar{A}, \bar{B}\}$. Suppose first that $m(\bar{A}, \bar{C})>1$. Then by $4.3, K=$ $\langle\bar{A}, \bar{C}\rangle=H M$ where $H=\left\langle C_{D}(u)\right\rangle, u=u(A, C)$, and $M=O_{p}(K) . \quad y$ fixes $A$ so $y$ fixes $\Gamma_{C \bar{A}}$ for $A \sim C$. As $\left|\Gamma_{C \bar{A}}\right|$ is a power of $p$ and $p \equiv$ $1 \bmod r, x$ fixes a point $C$ of $\Gamma_{C \bar{A}}$. As this holds for each $A \in \bar{A}$, we can assume $x$ normalizes $H$. Thus with $4.3, F(y u)=\{\bar{A}, \bar{C}\}$ and $[y$, $u]=1$. Now $J=I C_{J}(y)$, so $[M, y] \leqq M \cap I=[A, M]$ by 3.3. So if $y$ acts by scalar multiplication on $\bar{C}$, then $[M, y] \leqq[A, M] \cap[C, M]=$ $Z(K)$ by 3.3 , so that $y$ centralizes $M / Z(K)$. But $y$ does not even centralize $[A, M] / Z(K)$. So $y$ does not have scalar action on $\bar{C}$.

Set $\bar{E}=\bar{B}^{u}$. $y$ has scalar action on $\bar{E}$ and $\bar{B}$, so as above $m(\bar{E}$, $\bar{B})=1$. $\langle E, B\rangle \cong S L_{2}(q)$ or $L_{2}(q)$ so there exists an involution $t$ with cycle $(\bar{E}, \bar{B})$ inverting $y \bmod C(\bar{B})$. Thus $u t \in N(\bar{B})$ inverts $y \bmod C(\bar{B})$, while $N(\bar{B})=C(\bar{B}) C(y)$. So $|y C(\bar{B}) / C(\bar{B})|=|y I / y| \leqq 2$.

Assume $|y I / y|>2$. Then as above $m(\bar{E}, \bar{F})=1$ for all $\bar{E}, \bar{F} \in F(y)$ and $C_{G}(y)$ fixes $F(y)$ pointwise. Now if $z$ is an element centralizing $\bar{A}, \bar{B}$, and $y$ then $F(z)=\left\langle C_{D}(z)\right\rangle \cap \bar{D}$ and minimality of $G$ implies $F(z) \cap$ $F(y)=\{\bar{A}, \bar{B}\}$. Thus $z$ moves $\bar{C}$, so $z=1$. Now there exists an involution $t$ with cycle $(\bar{A}, \bar{B})$ inverting $y$ modulo $C(\bar{A}) \cap C(\bar{B})$. Thus $y^{t}=$ $y^{-1}$. Similarly there exists $s$ with cycle $(\bar{B}, \bar{C})$ inverting $y$. So ts 
moves $\bar{A}$ to $\bar{C}$ and centralizes $y$, a contradiction. Thus we have shown (3).

Assume the hypothesis of (4). Let $E \in \bar{A}$, and $C=\bar{B}(E)$. Then for $\alpha \in Q^{\sharp} \cap\langle A, C\rangle,\langle a\rangle \in \bar{A}$. So $\bar{A}=\left\{\langle a\rangle: a \in Q^{\sharp}\right\}$. Let $\Delta=\bar{A} \cup \bar{B}^{2}$.

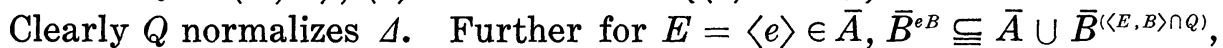
so as $\bar{A}=\left\{\langle a\rangle: a \in Q^{\sharp}\right\}, B$ normalizes $\Delta$. Thus $X=\langle\bar{A}, \bar{B}\rangle$ normalizes $\Delta$. Further $X^{A}$ is 2-transitive with $Q^{A} \unlhd X_{A}^{A}$ and regular on $\Delta-\{\bar{A}\}$. Therefore, a result [11] of Kantor and Seitz implies $X^{4} \cong L_{2}(q)$. This yields (4).

Assume the hypothesis of (5). Then there exists $y \in\langle A, C\rangle$ with $|y I / I|=4$ inducing scalar action on $Q \cap\langle A, C\rangle$ and $\langle\bar{B}\rangle \cap\langle A, C\rangle$. By (2), $x=y^{2}$ inverts $Q$ and $\langle\bar{B}\rangle$, so orbits of $x$ on $\bar{A}$ have order at most two. Suppose $\left(A_{1}, A_{2}\right)$ is such an orbit. Let $B_{2}=\bar{B}\left(A_{2}\right)$ and set $X=$ $\left\langle A_{1}, B_{2}\right\rangle$. Then $y$ normalizes $X$ with $x$ inverting $Q \cap X$, so $y$ induces scalar action on $Q \cap X$ and fixes $A_{1}$, a contradiction. Thus $y$ fixes $\vec{A}$ pointwise and induces scalar action on $Q$. This yields (5).

It remains to show (6). Assume $m(\bar{A}, \bar{C})>1$ and let $u=u(A, C)$. By $4.4, J=0(J) C_{J}(u)$. As $J=I C_{J}(y),[u, y] \leqq 0(I)$. Thus some conjugate $v$ of $u$ centralizes $y$. Now if $p>5$ or $p=5$ and $\langle A, E\rangle \cong L_{2}\left(5^{n}\right)$ or $S L_{2}\left(5^{n}\right), n$ even, for some $E \in \bar{B}$, then we can choose $y$ with $|I y / I|>2$. So by (3), $F(y)=\{\bar{A}, \bar{B}\}$. As $[v, y]=1$ and $v$ fixes $\bar{A}, v$ fixes $\bar{B}$. So $v$ centralizes some $B \in \bar{B}$, and by 4.3 , as $m(\bar{A}, \bar{B})=1, v \in I$. But this is impossible as $u \notin I$.

It follows from (4) that $\langle\bar{A}, \bar{B}\rangle \cong L_{2}(q)$ or $S L_{2}(q)$ with $q=p^{n}, n$ odd. So $\bar{A}=\left\{\langle a\rangle: a \in Q^{\sharp}\right\}$. But by 4.3 , $\langle\bar{A}, \bar{C}\rangle=H=\left\langle C_{D}(u)\right\rangle O_{p}(H)$ with $O_{p}(H) \neq Z(H)$. Thus there exists $a \in Q^{\sharp} \cap O_{p}(H)$ with $\langle a\rangle \notin \bar{A}$, a contradiction.

Lemma 4.6. $m(\bar{A}, \bar{B})=1$ for all $\bar{B} \neq \bar{A}$.

Proof. Assume not. Then by 4.5.6, $m(\bar{A}, \bar{B})>1$ for all $\bar{B} \neq \bar{A}$. Let $u=u(A, B), v=u(A, C)$. By $4.4, u$ is conjugate to $v$ under $J$, so $J$ takes $\bar{C}$ to a point of $F(u)$. But by 4.3 and $4.4, C_{G}(u)^{F(u)}$ is 2-transitive. Thus $J$ is transitive on $\bar{D}-\{\bar{A}\}$. Let $K=\langle\bar{A}, \bar{B}\rangle, H=$ $\left\langle C_{D}(u)\right\rangle$ and $M=O_{p}(K)$. Let $\Omega=\mathbf{U}_{K \cap J} C_{Q}\left(u^{k}\right)$. Suppose $w \in u^{J}$ inverts $1 \neq x \in \Omega$. Then $w u^{k}$ inverts $x$ while by $4.4, w u^{k}$ has odd order. So $X=\left[Q, u^{J}\right] \leqq\langle Q-\Omega\rangle \leqq M \cap Q$ by 3.3. But $X \unlhd J, J$ is transitive on $\bar{D}-\{\bar{A}\}$ and $M \cap Q$ fixes $\bar{B}$, so $X$ fixes $\bar{D}$ pointwise, contradicting 3.1.5.

Lemma 4.7. (1) There exists a prime $r$ such that for all $\bar{B} \neq \bar{A}$, $J=I N_{L}(R)$ for some r-group with $F(R)=\{\bar{A}, \bar{B}\}$.

(2) $G^{\bar{D}}$ is doubly transitive. 
Proof. (1) implies that there exists a prime $r$ such that for any $\bar{B} \neq \bar{A}$, a Sylow $r$-subgroup of $G_{\bar{A} \bar{B}}$ fixes only two points. This implies $G^{\bar{\nu}}$ is doubly transitive. So it suffices to proof (1). But unless $p=5$ there exists a prime $r$ dividing $p-1$ and an $r$-element $y \in\langle A, B\rangle$ fixing $\bar{A}$ and $\bar{B}$ with $|I y / I|>2$. So 4.5 implies (1) unless $p=5$ and $\langle\bar{A}, \bar{B}\rangle=H \cong L_{2}\left(5^{n}\right)$ or $S L_{2}\left(5^{n}\right), n$ odd. As $5^{n}=|Q|=|\langle\bar{A}\rangle|$, this holds for all $\bar{B} \neq \bar{A}$.

Suppose $u$ is an involution in $I$ and let $(\bar{C}, \bar{E})$ be a cycle in $u$ and $X=\langle\bar{C}, \bar{E}\rangle$. As $u$ does not centralize $X, u$ acts fixed point free on $X \cap \bar{D}$, so as $n$ is odd, $u$ induces an outer automorphism in $P G L_{2}\left(5^{n}\right)$ on $X$, and thus there exists a 2-element $y \in X$ inducing scalar action in $G F(5)$ on $\langle\bar{C}\rangle$ and $\langle\bar{E}\rangle$ with $y^{2}$ not centralizing $\langle\bar{C}\rangle$. Thus by 4.5 , $|F(y)|=2$, so $|\bar{D}|=m$ is even.

Assume $m$ is odd. Then $I$ has odd order. Let $x$ be the involution in $\langle A, B\rangle \cap J$. By 4.5, $J=I C_{J}(x)$. But as $m$ is odd $J$ contains a Sylow 2-subgroup of $G$, so the $Z^{*}$-theorem contradicts $O_{\infty}(G)=1$. Therefore, $m$ is even.

If a Sylow 2-subgroup of $G_{\bar{A} \bar{B}}$ fixes exactly two points for every $\bar{B} \neq \bar{A}$, then $G^{D}$ is doubly transitive. So choose $\bar{B}$ such that a Sylow group of $G_{\overline{A B}}$ fixes more than two points. Then $H=\langle\bar{A}, \bar{B}\rangle \cong L_{2}\left(5^{n}\right)$, $C_{J}(H)$ has odd order and the involution $x \in H_{\overline{A B}}$ fixes three or more points. Suppose $y^{2}=x$ for some $y \in G$. If $(\bar{C}, \bar{E})$ is a cycle of $y$ in $F(x)$ then $y$ normalizes $X=\langle\bar{C}, \bar{E}\rangle$ so as $y^{2}=x$ and $n$ is odd, $y$ fixes two points in $X \cap \bar{D}$, which must be $\bar{C}$ and $\bar{E}$. This is a contradiction, so $x$ is not rooted in this manner.

Suppose $I$ has odd order. Then by $4.5, J=I C_{J}(y)$ for any involution $y \in\langle\bar{A}, \bar{C}\rangle$ and any $\bar{C} \neq \bar{A}$. So $y \in x^{I}$. Let $u$ be an involution. We may assume $u$ has cycle $(\bar{A}, \bar{B})$. So $u$ normalizes $H$, and as $I$ has odd order and $x$ is not rooted in $\langle u, H\rangle, u \in H$. Thus $u \in x^{G}$. Thus $G$ has one class of involutions, so as $x$ is not rooted, a Sylow 2-subgroup of $G$ is elementary abelian. Walter's classification of such groups [13] implies $G \cong L_{2}\left(5^{n}\right)$, a contradiction. So $I$ has even order. Thus $x$ centralizes some involution $u \in I$; as $|\bar{D}|$ is even, there exists $\bar{R} \in F(x) \cap F(u)-$ $\{\bar{A}\}$; minimality of $G$ implies $\left\langle C_{\bar{D}}(u)\right\rangle \cong L_{2}\left(5^{n}\right), S L_{2}\left(5^{n}\right)$ or $U_{3}\left(5^{n}\right)$, so $F(x) \cap F(u)=\{\bar{A}, \bar{R}\}$.

Consider $C_{G}(x)^{F(x)}$. Arguments such as in 4.5.3 and in the last paragraph show that nontrivial elements of $C(x)^{F(x)}$ fix at most two points. Let $(\bar{C}, \bar{E})$ be a cycle of $u$ in $F(x)$. We have shown $x$ is rooted modulo $C(\bar{C}) \cap C(\bar{E})$, while $x$ is not rooted. So $C(\bar{C}) \cap C(\bar{E})$ has even order and there exists an involution $v \in C(x)^{F(x)}$, fixing $\bar{C}$ and $\bar{E}$, and centralizing $u$. $v$ acts on $F(x) \cap F(u)=\{\bar{A}, \bar{R}\}$. Let $L=C C_{\overline{A R}(x)}^{F(x)} \quad L$ acts semiregularly on $F(x)-\{\bar{A}, \bar{R}\}$ and $C_{L}(v)$ acts on $F(v) \cap F(x)=$ $\{\bar{C}, \bar{E}\}$, so $\langle v\rangle=C_{L}(u)$. So a Sylow 2-subgroup $S$ of $\langle L, v\rangle=L^{*}$ is semidihedral or dihedral, and there are one or two classes of involu- 
tions in $L^{*}-L$, respectively. But if $\bar{T} \in F(x)-\{\bar{A}, \bar{R}\}$ let $t$ be the involution in $C(x)^{F(x)}$ fixing $\bar{T}$ and $\bar{T}^{u}$ and centralizing $u$. Then $t \in$ $v_{i}^{L}, i=1$ or 2 , one of the (at most) two classes of involutions in $L^{*}$ $L$. So $L$ takes $F(t) \cap F(x)=\left\{\bar{T}, T^{u}\right\}$ to $F(x) \cap F\left(v_{i}\right)$. Thus $L$ has one orbit, or two orbits of equal length, on $F(x)-\{\bar{A}, \bar{R}\}$, for $S$ semidihedral or dihedral, respectively. It now follows easily that $C(x)^{F(x)}$ is 2transitive. But $J$ and therefore $C_{J}(x)$ cannot take $\bar{B}$ to $\bar{R}$ as there is no involution in $I$ fixing $\bar{B}$. This last contradiction completes the proof of 4.7.

Set $L=G_{\bar{A} \bar{B}}, H=\langle\bar{A}, \bar{B}\rangle, K=C_{G}(H)$, and $Q=\langle\bar{A}\rangle$.

LEMma 4.8. (1) $J=I L$ and $K \neq 1$.

(2) $H \cong L_{2}(q)$ or $S L_{2}(q)$.

Proof. By 4.7.1 there exists a prime $r$ such that a Sylow $r$ subgroup $R$ of $L$ fixes only $\bar{A}$ and $\bar{B}$, and $J=I N_{J}(R) . N_{J}(R)$ acts on $F(R)=\{\bar{A}, \bar{B}\}$; so $N_{J}(R) \leqq L$. If $K=I \cap L=1$ then $I$ is regular on $\bar{D}-\{\bar{A}\}$ by 4.7.2, so [11] implies $G \cong L_{2}(q)$ or $U_{3}(q)$. Thus $K \neq 1$. Minimality of $G$ implies $H=\left\langle C_{D}(K)\right\rangle \cong S L_{2}(q)$ or $L_{2}(q)$.

Lemma 4.9. Suppose $x \in L^{\sharp}$ with $\left|C_{Q}(x)\right|=q_{0}>1$. Then $\left\langle C_{D}(x)\right\rangle \cong$ $L_{2}\left(q_{0}\right), S L_{2}\left(q_{0}\right)$ or $U_{3}\left(q_{0}\right)$ and $|F(x)|=q_{0}+1$ or $q_{0}^{3}+1$.

Proof. Minimality of $G$ yields the desired form for $\left\langle C_{D}(x)\right\rangle$. If $\bar{C} \in F(x)$ then $[x, C]=1$ where $C=\bar{C}(A), A \in C_{\bar{A}}(x)$. Thus $|F(x)|=q_{0}+$ 1 or $q_{0}^{3}+1$.

Lemma 4.10. Set $n=|\bar{D}|$. Then $(n-1,|K|)$ is a power of $p$.

Proof. Let $r$ be a prime divisor of $|K|$, and $R$ a Sylow $r$-subgroup of $K$. By 4.9, $F(R)=q+1$ or $q^{3}+1$, so if $r \neq p$ then a Sylow $r$ subgroup $R_{1}$ of $N_{I}(R)$ fixes a second point $\bar{B}$ of $F(R)$; that is $R_{1}=R$. So $R$ is Sylow in $I$ and $r$ does not divide $n-1=|I: K|$.

LEMMA 4.11. $|\bar{D}|=n$ is even. If $u$ is an involution then $n \equiv$ $|F(u)| \bmod 4 . \quad|L|$ is even.

Proof. Results of Bender on doubly transitive groups [5.6] imply $L$ has even order. By 3.1, $G$ is simple, so any involution $u$ must act as an even permutation on $\bar{D}$. Thus $n \equiv|F(u)| \bmod 4$. If $n$ is odd, 2-elements fix an odd number of points. So by 4.8 and $4.9,|K|$ and $|L / H K|$ are odd. And by 4.5.3, $|H \cap L| \neq 0 \bmod 4$. As $L$ has even order, $|H \cap L| \equiv|L| \equiv 2 \bmod 4$. Thus $p \equiv q \equiv 5 \bmod 8$. Let $u$ be the involution in $H \cap L$, and $S$ a $u$-invariant Sylow 2-subgroup of $I$. As 
$n$ is odd and $J=I L, S\langle u\rangle$ is Sylow in G. As $G$ has no subgroup of index two, $S \neq 1$. Let $s$ be an involution in $S$, and $(\bar{B}, \bar{C})$ a cycle in $s$. Then $s$ normalizes $X=\langle\bar{B}, \bar{C}\rangle$ and as $|F(s)|=1, s$ acts fixed point free on $\bar{D} \cap X$. So as $p \equiv q \equiv 5 \bmod 8,\langle s, X\rangle \cong P G L_{2}(q)$ and there exists $y \in\langle s, X\rangle$ of order 4 inducing scalar multiplication on $\langle\bar{B}\rangle$ and fixing $\bar{B}$ and $\bar{C}$. By 4.5.3, $|F(y)|=2$, contradicting $n$ odd.

Lemma 4.12. If $J=O(I) L$ then $J=O_{\pi}(I) L$, where $\pi$ is the set of primes dividing $n-1$. Also $O_{p}(K) \neq 1$, and $O_{\pi}(I)$ is not nilpotent.

Proof. Set $P=O_{\pi}(I)$. If $P \neq O(I)$ let $R / P$ be minimal normal in $J / P, R<O(I) . \quad R / P$ is an $r$-group for some prime $r$ and by a Frattini argument, $J=P N_{J}\left(R_{1}\right)$ where $R_{1}$ is a Sylow $r$-subgroup of $R$ contained in $K$. By $4.9, N_{J}\left(R_{1}\right)=L P_{1}$ where $\left|P_{1}\right|=q$ or $q^{3}$, and $P_{1} \unlhd N_{J}\left(R_{1}\right)$. Thus $P P_{1} \unlhd J$, so $P_{1} \leqq P$ and $J=P L$. Results of Kantor and Seitz on doubly transitive groups $[11,12]$ imply $P$ is not nilpotent or regular on $\bar{D}-\{\bar{A}\}$. Thus $1 \neq P \cap L=P \cap K=O_{p}(K)$ by 4.10 .

Lemma 4.13. Let $X \subseteq L$ fix 3 or more points of $\bar{D}$. Then $C_{G}(X)^{F(X)}$ is doubly transitive.

Proof. It suffices to show there exists a prime $r$ such that a Sylow $r$-subgroup of $C_{L}(X)$ fixes only $\bar{A}$ and $\bar{B}$. Thus with 4.5 we can assume $q=5^{m}$ with $m>1$ odd. Thus there is an $r$-element $1 \neq$ $y \in H \cap L, r>2$, and as $m$ is odd $y$ is not inverted in $J / I$ by 4.8 . Thus arguing as in $4.5, F(y)=\langle\bar{A}, \bar{B}\rangle . \quad[y, X]=1$ unless $C_{Q}(X) \neq 1$, in which case 4.9 implies $C_{G}(X)^{F^{(}(X)}$ is doubly transitive.

Lemma 4.14. Assume $q \equiv-1 \bmod 4$ and $x$ is an involution in $L$ inverting $Q$ with $|F(x)|>2$. Then $|F(x)|=q+1$.

Proof. As $q \equiv-1 \bmod 4, q$ is an odd power of $p$, so no element in $H \cap L$ is inverted in $J / I$. Thus if $y \in H \cap L$ with $|y|>2$ then $|F(y)|=2$. Therefore, with 4.9 and $4.13, C_{G}(x)^{F(x)}$ is a Zaussenhaus group. So $C_{G}(x)^{F(x)}$ has a normal subgroup isomorphic to $L_{2}(m)$, of index at most two, with $|F(x)|=m+1$. Now if $m \equiv 1 \bmod 4$ then by 4.9 and $4.11, K$ has odd order, and $\langle x\rangle$ is Sylow in $L$, so that $\left|C_{L}(x)^{F^{(x)}}\right|$ is odd, contradicting $m \equiv 1 \bmod 4$. So $m \equiv-1 \bmod 4$. Thus $C_{L}(x)^{F(x)}$ is cyclic and inverted by any $t \in C_{G}(x)$ with cycle $(\bar{A}, \bar{B})$. As we can choose $t \in H$, and $[K, t]=1$, it follows that $\left|C_{K}(x)\right|=\varepsilon \leqq 2$. Further $\varepsilon(m-1) / 2=\left|C_{L}(x)^{F(x)}\right|=\varepsilon|H \cap L|=\varepsilon(q-1) / 2$, so $m=q$.

Lemma 4.15. Suppose $u$ is an involution in $Z^{*}(L)$ fixing 3 or more points. Then $u \in Z^{*}(J)$. 
Proof. $u \in Z^{*}(L)$ so $u^{L} \cap C_{L}(u)=\{u\}$. Now 4.13 implies $u^{G} \cap L=u^{L}$. Further as $|\bar{D}|$ is even, if $v$ is a conjugate of $u$ in $J$ centralizing $u$ then we can assume $v \in L$, so $v \in u^{G} \cap C_{L}(u)=u^{L} \cap C_{L}(u)=\{u\}$. Thus by the $Z^{*}$-theorem, $u \in Z^{*}(J)$.

LEmmA 4.16. If $H \cong L_{2}(q)$ then $H \cap \bar{D}=F(X)$ for any $1 \neq X \leqq K$.

Proof. If $F(X) \neq H \cap \bar{D}$ then by $4.9, H \leqq\left\langle C_{D}(X)\right\rangle \cong U_{3}(q)$, so $H \cong S L_{2}(q)$.

LEMma 4.17. Assume $u$ is an involution in $L$ fixing $m+1 \geqq 3$ points, let $c=\left|L: C_{L}(u)\right|$ and let $e$ be the number of conjugates of $u$ with cycle $(\bar{A}, \bar{B})$. Then $|D|-1=m(m+1) e / c+m$.

Proof. Let $\Omega$ be the set of pairs $(v, \alpha)$ where $v \in u^{G}$ and $\alpha$ is a cycle in $v$. Then $\left|u^{G}\right|(n-m-1) / 2=|\Omega|=n(n-1) e / 2$ where $n=|\bar{D}|$. Further by $4.13,\left|u^{G}\right|=n(n-1) c / m(m+1)$.

LemMA 4.18. (1) Let $S$ be a 2-group such that $C_{Q}(S) \neq 1$. Then $S$ has rank at most one.

(2) $J=O(I) L$.

Proof. Suppose $1 \neq\langle u\rangle=H \cap L$. Then by 4.15, $u \in Z^{*}(I)$, so $J=O(I) L$. Define $P=O_{\pi}(I)$ as in 4.12, and assume $S$ has 2-rank at least two. Then $P=\Pi_{s^{\sharp}} C_{P}(s)$, while by $4.9, C_{P}(s)$ is a $p$-group for $s \in S^{\sharp}$. Thus $P$ is a $p$-group, contradicting 4.12 .

So $H \cap L=1$ and by $4.16, N_{I}(H)=Q K$ is strongly embedded in I. As $Q \leqq O(I)$ and $[K, H \cap L]=1$, Bender's classification of groups with a strongly embedded subgroup [6] implies $J=O(I) N_{J}(H \cap L)$. By 4.5, augmented by arguments such as in 4.13 for the case $q=5^{m}$, $m$ odd, $N_{J}(H \cap L)=L$. Now arguing as above, $S$ has 2-rank at most one.

Define $P=O_{\pi}(I)$ as in 4.12. Set $P_{0}=O_{p}(K) . \quad P_{0} \neq 1$ by 4.12 and 4.18.

Lemma 4.19. (1) $F(X)=H \cap \bar{D}$ for $1 \neq X \leqq P_{0}$.

(2) $H \cap K=1$.

(3) Assume $u$ is an involution in $K$ and let $v \in u^{G}$ have cycle $(\bar{A}, \bar{B})$. Let $P_{1}$ be $a\langle u, v\rangle$ invariant Sylow p-group of $O(K)$. Then $\left[v, P_{1}\right]=P_{1}$ and $\left[u, P_{1}\right] \neq 1$.

Proof. Assume $1 \neq X \leqq P_{0}$ with $F(X) \neq H \cap D$. Then $Y=$ $\left\langle C_{D}(X)\right\rangle \cong U_{3}(q)$ by 4.9. So $H \cap K=\langle u\rangle \neq 1$. Further as $N_{K}(X)^{F(X)}$ is a $p^{\prime}$-group, $X=P_{0}$. Let $(\bar{C}, \bar{E})$ be a cycle in $u$ and $v \in u^{G}$ fix $\bar{C}$ and $\bar{E}$. Then $[u, v]=1$ so $v$ acts on $\left\langle C_{D}(u)\right\rangle=H$ and thus also on $P_{0}$. 
$v$ induces an automorphism on $Y \cong U_{3}(q)$ and therefore fixes points $\bar{A}_{i} \in F\left(P_{0}\right)$. So $\bar{C} \in\left\langle\bar{A}_{1}, \bar{A}_{2}\right\rangle \leqq Y$ and therefore $F\left(P_{0}\right)=\bar{D}$, a contradiction. This yields $(1)$.

Assume $1 \neq\langle u\rangle=H \cap K$. Then in particular $\left[u, P_{0}\right]=1$. Let $v \in u^{G}$ have cycle $(\bar{A}, \bar{B})$. $\quad v$ acts on $P_{0}$ and $F(v) \cap F(x)=F(v) \cap F(u)=$ $\varnothing$ for $x \in P_{0}^{\sharp}$. Thus $C_{P_{0}}(v)$ acts fixed point free on $F(v)$ of order $q+$ 1 , so $C_{P_{0}}(v)=1$. Define $e$ and $c$ as in 4.17. It follows that $c=1$ and $e \equiv 0 \bmod p$. So by $4.17,|\bar{D}|-1=q[(q+1) e / c+1] \equiv q \bmod p q$. So $P_{0} Q$ is Sylow in $P$ and $u$ centralizes $P_{0} Q$, and inverts a Hall $p^{\prime}$-group $P_{1}$ of $P$. Thus $P=P_{1} \times\left(P_{0} Q\right)$ is nilpotent, contradicting 4.12. This yields (2).

Assume the hypothesis of (3) and define $c$ and $e$ as in 4.17. Arguing as above, $\left[v, P_{1}\right]=P_{1}$, so $p$ divides $e$. By $4.18, L=O(K) C_{L}(u)$, so if $\left[P_{1}, u\right]=1$, then $p$ does not divide $c$. But then arguing as above we have a contradiction.

\section{LEMMA 4.20. $q \equiv 1 \bmod 4$.}

Proof. Assume $q \equiv-1 \bmod 4$. By 4.9, 4.10, and 4.14, $C_{P}(x)$ is a $p$-group for any involution $x \in L$, while by $4.12, P$ is not a $p$-group. Thus $L$ has 2-rank one. Suppose $K$ has odd order. By 4.11, $L$ has even order so there exists an involution $x \in L$ and $\langle x\rangle$ is Sylow in $J$. If $|F(x)|=2$, then by 4.11, $n=|\bar{D}| \equiv 2 \bmod 4$, and [2] implies $G \cong$ $L_{2}(q)$. Thus by 4.14, $|F(x)|=q+1$. Let $v$ be a conjugate of $x$ with cycle $(\bar{A}, \bar{B})$. We may choose $v=t$ or $t x$ where $t \in H$. By $4.16, F\left(P_{0}\right)=$ $H \cap \bar{D}$, so $\left|F\left(P_{0}\right) \cap F(v)\right|=0$ or 2 . Thus if $C_{P_{0}}(v) \neq 1$ then $1 \equiv q+$ $1=|F(x)| \equiv 0$ or $2 \bmod p$, so $v$ inverts $P_{0}$. Thus $v=t x$, and $x$ inverts $P_{0}$. Define $e$ and $c$ as in 4.17. Then $e=(q-1) c / 2$, so by $4.17, n-$ $1=q\left(q^{2}+1\right) / 2$. In particular $Q P_{0}$ is Sylow in $P$ and inverted by $x$. As $|F(x)|=q+1, x$ inverts an $x$-invariant Sylow $r$-subgroup of $P$ for $r \neq P$, with 4.10. Thus $x$ inverts $P$, and $P$ is abelian, contradicting 4.12 .

So $K$ contains an involution $u$. Let $v \in u^{G}$ have cycle $(\bar{A}, \bar{B})$, with $[v, u]=1$. As $H \cap K=1$ and $v$ acts fixed point free on $F(u)=H \cap$ $\bar{D}, v=t$ or $u t$ where $t \in H$. By $4.19\left[v, P_{0}\right] \neq 1$, so $v=u t$. Thus defining $e$ and $c$ as in 4.17, $e=(q-1) c / 2$, so by $4.17, n-1=q[(q+$ 1) $e / c+1]=q\left(q^{2}+1\right) / 2$. Let $R$ be a $\langle u\rangle(H \cap L)$ invariant $r$-Sylow group of $P$, where $r \neq p$. Then $\langle u\rangle(H \cap L)$ acts semiregularly on $R$, $|R|>q$. As a $p^{\prime}$-Hall group of $P$ has order $\left(q^{2}+1\right) / 2,\left(q^{2}+1\right) / 2$ is a prime power. Thus $q$ is a prime (e.g. Lemma 3.1, [1]). $P_{0}$ acts semiregularly on $\bar{D}-F\left(P_{0}\right)$ of order $q\left(q^{2}+1\right) / 2-q=q\left(q^{2}-1\right) 2$, so $\left|P_{0}\right|=$ $q$. Thus $Q=C_{P}(u) \leqq Z(P)$, or $[P, u]$ is a Hall $p^{\prime}$-group of $P$. In either event $P$ is nilpotent, contradicting 4.12 . 


\section{LEMma 4.21. $|K|$ is odd.}

Proof. Assume $K$ has even order and let $u$ be an involution in $K$ and $v$ a conjugate of $u$, centralizing $u$, with cycle $(\bar{A}, \bar{B})$. By 4.1, $\left[v, P_{1}\right]=P_{1}$ and $\left[u, P_{1}\right] \neq 1$. So $C_{P_{1}}(u v) \neq 1,|F(u v)| \equiv 0 \bmod p$ and $u v \notin u^{G}$. So by 4.11 and 4.18, $u v \in x^{G}$ or $(u x)^{G}$ where $x \in H$. Now $\left[x, P_{0}\right]=1$ so $|F(x)| \equiv 2 \bmod p$. Thus $u v \in(u x)^{G}$ and as $|F(u v)| \equiv 0$ $\bmod p$ and $\left|F\left(P_{0}\right) \cap F(u x)\right|=2, C_{P_{0}}(u x)=C_{P_{0}}(u)=1$. So $Q=C_{P}(u)$, yielding a contradiction as in 4.20 .

\section{LEMMA 4.22. L has 2-rank one.}

Proof. Assume not. Then as $|K|$ is odd by 4.21, there exists an involution $x \in H \cap L$ and an involution $u \in L$ with $\left|C_{Q}(u)\right|=r, q=r^{2}$, and $Q=C_{Q}(u) \times C_{Q}(u x)$. Notice $P=C_{P}(x) C_{P}(u) C_{P}(u x)=C_{P}(x) Q$. Set $m+1=|F(x)|$. As $P_{0}$ acts semi-regularly on $F(x)-\{\bar{A}, \bar{B}\}, m \equiv 1$ $\bmod p$. Let $P_{2}$ be a subgroup of $C_{P}(x)$ maximal with respect to being normal in $C_{J}(x)$ and semiregular on $F(x)-\{\bar{A}\}$. Let $M / P_{2}$ be a minimal subgroup of $C_{J}(x) / P_{2}$ contained in $C_{P}(x)$. By $4.10, M / P_{2}$ is a $p$-group and as $P_{2}$ is semi-regular on $F(x)-\{\bar{A}\}$ of order $m \equiv 1 \bmod p, P_{2}$ is a $p^{\prime}$-group. Thus $M=P_{2}\left(P_{0} \cap M\right)=P_{2} M_{0}$ and $C_{J}(x)=P_{2}\left(N\left(M_{0}\right) \cap\right.$ $\left.C_{J}(x)\right)=P_{2} C_{L}(x)$ as $F(x) \cap F\left(M_{0}\right)=\{\bar{A}, \bar{B}\}$. So $\left|P_{2}\right|=m$ and $P_{2} \leqq$ $Q C_{P}(x)=P$. Thus $P_{2} Q$ is regular on $\bar{D}-\{\bar{A}\}$. As $u$ inverts $P_{2}, P_{2} Q$ is nilpotent and thus contained in Fit $(P)$, the Fitting subgroup of $P$. So Fit $(P)$ is transitive on $\bar{D}-\{\bar{A}\}$ and nilpotent, contradicting 4.12.

\section{Lemma 4.23. $|\bar{D}| \equiv 2 \bmod 4$.}

Proof. Assume not. Let $x$ be the involution in $H \cap L$. By 4.11, $|F(x)| \equiv 0 \bmod 4$. As in 4.14, $C_{G}(x)^{F(x)}$ is a Zassenhaus group and $t$ inverts $L^{F(x)}$ where $t \in H$ has cycle $(\bar{A}, \bar{B})$. But $\left[t, P_{0}\right]=1$ and $P_{0} \cong$ $P_{0}^{F(x)}$, a contradiction.

4.22 and 4.23 together with [2] imply $G \cong L_{2}(q)$ or $U_{3}(q)$. Thus the proof of Theorem 4.1 is complete.

\section{Examples.}

Hypothesis 5.1. Let $V$ be a $2 m$ dimensional space over $G F(q), q$ a power of the odd prime $p$, with nondegenerate skew symmetric bilinear form (,). For $u \in V^{\sharp}$ the transvection $u^{*}$ determined by $u$ is the map

$$
u^{*}:\langle x\rangle \longrightarrow\langle x+(x, u) u\rangle
$$


considered as a projective transformation of $V$. Let $D=\left\{\left\langle u^{*}\right\rangle: u \in V^{\sharp}\right\}$ and $G=\langle D\rangle$.

$G$ is the $2 m$ dimensional projective symplectic group $S P_{2 m}(q)$ over $G F(q)$.

Lemma 5.2. Assume hypothesis 5.1. Let $A=\left\langle a^{*}\right\rangle$ and $B=\left\langle b^{*}\right\rangle$ lie in $D$ with $[A, B] \neq 1$. Set $L=\left\langle D_{A} \cap D_{B}\right\rangle$. Then

(1) $D$ is a class of p-transvections of $G$.

(2) $L / Z(L) \cong S P_{2 m-2}(q)$ for $m>1$.

Proof. Let $\left\langle c^{*}\right\rangle=C \in D$. Then $[A, C]=1$ if and only if $(a, c)=$ 0 . So (,) restricted to $\langle a, b\rangle$ is a nondegenerate skew symmetric bilinear form and therefore $\langle A, B\rangle$ is a homomorphic image of a subgroup of $S L_{2}(q)$. This yields (1). Similarly $L$ acts as a symplectic group on $\langle a, b\rangle^{\perp}$ yielding (2).

Hypothesis 5.3. Let $V$ be a n-dimensional vector space over $G F\left(q^{2}\right)$ with nondegenerate semibilinear form (,). For nonsingular vector $u$ let $u^{*}$ be the transvection determined by u considered as a projective transformation of $V$. Let $D=\left\{u^{*}:(u, u)=0\right\}$, and $G=\langle D\rangle$.

$G$ is the $n$ dimensional projective special unitary groups, $U_{n}(q)$.

Lemma 5.4. Assume hypothesis 5.3. Let $A=\left\langle a^{*}\right\rangle$ and $B=\left\langle b^{*}\right\rangle$ lie in $D$ with $[A, B] \neq 1$. Set $L=\left\langle D_{A} \cap D_{B}\right\rangle$ then

(1) $D$ is a class of p-transvections of $G$.

(2) $L / Z(L) \cong U_{n-2}(q)$ for $n \geqq 4$.

(3) $G$ contains a unique class of $D$-subgroups $K^{G}$ with $K / Z(K) \cong$ $U_{n-1}(q)$.

Proof. The proofs of (1) and (2) are as in 5.2. Assume $K$ is a $D$-subgroup of $G$ with $K / Z(K) \cong U_{n-1}(q)$. As $\left[a^{*}, c^{*}\right]=1$ if and only if $(a, c)=0,\left\langle u:\left\langle u^{*}\right\rangle \in K \cap D\right\rangle$ is a nonsingular hyperplane of $V$ preserved by $K$. As $G$ is transitive on such hyperplanes, (3) follows.

6. Proof of main theorem. For the remainder of this paper $G$ is a counter example of minimal order to the main theorem. Lemma 3.1 implies:

Lemma 6.1. $G$ is simple.

Theorem 4.1 implies: 


\section{LEMma 6.2. $\mathscr{D}(D)$ is connected.}

Let $A \in D$. By 2.4, $A$ is contained in a unique maximal set of imprimitivity $\alpha$ of $G^{D}$. Set $H=\left\langle D_{\alpha}\right\rangle, M=O_{\infty}(H)$, and $\Omega=\alpha^{G}$. By 2.4, $H$ is $D_{\alpha}^{*}$-simple. Minimality of $G$ implies $H / M \cong S p_{n}(q)$ or $U_{n}(q)$, for some power $q$ of $p$.

Lemma 6.3. Let $\beta \in D_{\alpha}, \gamma \in D_{\beta} \cap A_{\alpha}$. Set $\Gamma=D_{\alpha} \cap D_{\gamma}$ and $L=$ $\langle\Gamma\rangle$. Then $L M=H, M \neq Z(H)$ and $\alpha * \beta=\{\alpha\} \cup \beta^{M}$.

Proof. Let $B \in \beta$. $H / M \cong S p_{n}(q)$ or $U_{n}(q)$ has $V_{B M / M}$ as a set of imprimitivity on $D_{\alpha}^{*} M / M$, so $\langle\beta\rangle$ is abelian. Set $K=\left\langle D_{\beta} \cap \Gamma\right\rangle, H_{1}=$ $\left\langle D_{\beta}\right\rangle$, and $M_{1}=O_{\infty}\left(H_{1}\right)$.

Assume $n \geqq 4$. Then by 5.2 and $5.4, K M_{1} / M_{1} \cong U_{n-2}(q)$ or $S p_{n-2}(q)$. Suppose $L$ is not $D$-simple. Then by $2.1, L$ is the central product of two $D$-subgroups $L_{i}$. Let $B \in L_{1} . \quad K$ is $D$-simple, so $K=L_{2}$. Thus $\beta=B^{\perp} \cap L_{1}$, so $\mathscr{D}\left(L_{i} \cap D\right)$ is disconnected. Thus $L / O_{\infty}(L) \cong L_{2}(q) \times$ $L_{2}(q)$ or $U_{3}(q) \times U_{3}(q)$. As $U_{5}(q)$ contains no $D$-subgroup of the latter type, that case is eliminated. As $\beta=B^{\perp} \cap L_{1}, \beta=B^{\perp} \cap D_{\alpha}^{*}$. Now let $C \in \gamma$ with $X=\langle A, C\rangle \cong S L_{2}(q)$, and $x \in X$ fix $\alpha$ and $\gamma$ with $|x| \geqq 4$. $x$ centralizes $L$ and normalizes $H$. Suppose $L \neq\left\langle C_{D_{\alpha}^{*}}(x)\right\rangle=Y$. Then there exists $\delta \in A_{r} \cap Y$. Minimality of $G$ implies $\mathscr{D}(Y \cap D)$ is connected so we can choose $\delta \in D_{\sigma}$ for some $\sigma \subseteq L$. Let $Z=\langle\lambda, \delta\rangle$. As $\gamma, \delta \in$ $D_{o}, Z / O_{p}(Z) \cong S L_{2}(q)$. So as $[x, \delta]=1$, we get $[x, \lambda]=1$, a contradiction. So $L=Y$ and as $x$ induces an automorphism on $H / M \cong S p_{4}(q)$ or $U_{4}(q)$ with $Y / O_{\infty}(Y) \cong L_{2}(q) \times L_{2}(q)$, this automorphism has order two. As $|x|>2,1 \neq x^{2}$ centralizes $H / M$. As $\left[x^{2}, B^{\perp} \cap D_{\alpha}^{*}\right]=1,\left[H, x^{2}\right]$, so $\left\langle x^{2}\right\rangle=Z(X)$ and $X \cong S L_{2}(5)$. But now $C_{D}\left(x^{2}\right)$ is a component of $\mathscr{D}(D)$, contradicting 6.2.

So $L$ is $D$-simple. Therefore, minimality of $G$ implies $L / O_{\infty}(L) \cong$ $H / M$ and $O_{\infty}(K)=M_{1} \cap K \neq Z(K)$. As $D_{r} \cap\left(\alpha^{*} \beta\right)=\{\beta\}, \alpha^{*} \beta=\{\alpha\} \cup \beta^{M}$.

Thus we may assume $n \leqq 3$. Suppose $X=\langle A, E\rangle \cong S L_{2}(q)$ for $E \in D_{\beta}^{*}$. Then we may choose $C \in \gamma \cap X$. Let $\langle u\rangle=Z(X)$. Then $u \in$ $\langle A, C\rangle$, so $[u, L]=1$. $u$ acts on $H / M$ and centralizes $\beta$, so $J=$ $\left\langle C_{D_{\alpha}^{*}}(u)\right\rangle$ contains a $D$-subgroup isomorphic to $S L_{2}\left(q_{0}\right)$ for some $q_{0}$ dividing $q$. Let $\langle v\rangle$ be the center of that subgroup. If $J \neq L$ then considering $\langle J, X\rangle$, minimality of $G$ yields a contradiction. So $J=L$ and $[v, X]=1$. $\left\langle C_{D_{\alpha}^{*}}(v)\right\rangle=X_{0} \cong S L_{2}(q)$, so arguing on $v$ in place of $u$ we get $X_{0}=L$ and $q_{0}=q$. If $H=L M$ then as $D_{\alpha} \neq D_{\gamma}, M \neq Z(H)$, and as above $\alpha^{*} \beta=\{\alpha\} \cup \beta^{M}$. So we may assume $H / M \cong U_{3}(q)$. Define $x$ as above with $u \in\langle x\rangle$. $[x, L]=1$ and $x$ acts on $H / M \cong U_{3}(q)$, so as $2<|x|$ divides $q-1, u \in\langle x\rangle$ centralizes $H / M$, contradicting $L M \neq H$.

So $X$ does not exist. Thus $H \cong L_{2}(q)$. Claim $\beta=B^{\perp} \cap D_{\alpha}^{*}=$ $\alpha^{*} \beta-\{\alpha\}$. For if not $\beta \cong\left\langle\alpha^{*} \beta-\{\alpha, \beta\}\right\rangle$ whereas $\alpha \nsubseteq\left\langle\alpha^{*} \beta-\{\alpha, \beta\}\right\rangle$. 
Choose $1 \neq x \in H_{1}$ fixing $\alpha$ and $\lambda$. $x$ acts on $H$ and centralizes $\beta$, so $[x, H]=1$. Let $E \in D_{\alpha}^{*}-L$ and $C \in \gamma$. The action of $x$ on $\langle C, E\rangle$ yields a contradiction.

Lemma 6.4. Let $(\alpha, \gamma, \beta)$ be a triangle in $\Omega$. Then there exists $\sigma$ with $\alpha, \beta$, and $\gamma$ in $D_{\sigma}$.

Proof. Claim $\mathscr{D}(\Omega)$ has diameter two. For if not $\alpha \beta \gamma \delta$ be a chain with $d(\alpha, \delta)=3$. Let $H_{1}=\left\langle D_{\gamma}\right\rangle, M_{1}=O_{\infty}\left(H_{1}\right), \Gamma=D_{\alpha} \cap D_{\gamma}$ and $L=\langle\Gamma\rangle$. Then by $6.3, H_{1}=L M_{1}$, so $\delta M_{1}=\sigma M_{1}$ for some $\sigma \in \Gamma$. Thus $\sigma \in D_{\alpha} \cap D_{\delta}$, contradicting $d(\alpha, \delta)=3$. Thus $\mathscr{D}(\Omega)$ has diameter two, so if $(\alpha, \gamma, \beta)$ is a triangle, by $6.3, L M=H$. So again there exists $\sigma \in \Gamma$ with $\sigma M=\beta M . \quad \alpha, \beta$, and $\gamma$ are in $D_{\sigma}$.

Lemma 6.5. Let $\gamma \in A_{\alpha}$. Then $\langle\alpha, \gamma\rangle \cong S L_{2}(q)$ and $|\langle\alpha\rangle|=q$.

Proof. Set $X=\langle\alpha, \gamma\rangle$. By 6.4, there exists $\beta \in D_{\alpha} \cap D_{r}$. Let $H_{1}=\left\langle D_{\beta}\right\rangle, M_{1}=O_{\infty}(H)$. Suppose $A \neq E \in \alpha$ with $A \equiv E \bmod M_{1}$. Then $A=\langle a\rangle, E=\langle e\rangle$ with $x=a e^{-1} \in M_{1}$. Thus $x$ fixes every singular line $\beta^{*} \delta=\{\beta\} \cup \delta^{M_{1}}$ through $\beta$. As $H \leqq C_{G}(x)$ is transitive on $D_{\alpha}, x$ fixes all singular lines through any $\beta \in D_{\alpha}$. Let $\sigma \in A_{\alpha}$. By 6.3, there are distinct singular lines $\beta_{i}^{*} \sigma, i=1,2$, with $\beta_{i} \in D_{\alpha}$. Then $x$ fixes $\left(\beta_{1}^{*} \sigma\right) \cap$ $\left(\beta_{2}^{*} \sigma\right)=\{\sigma\}$. Thus $x$ fixes $\Omega$ pointwise. But this contradicts 6.1.

So $|\langle\alpha\rangle|=|\langle\alpha\rangle M / M|=q$ by 6.3. By $6.3, X / O_{p}(X) \cong S L_{2}(q)$, so $|\langle\alpha\rangle|=q, O_{p}(X)=1$.

LEMMA 6.6. $\Omega$ is locally conjugate in $G,\left\langle\alpha^{\perp}\right\rangle$ is transitive on $A_{\alpha}$, and $G^{2}$ is rank 3 .

Proof. By 6.5, $\Omega$ is locally conjugate in $G$. Therefore, to show $\left\langle\alpha^{\perp}\right\rangle$ is transitive on $A_{\alpha}$ and thus that $G^{\alpha}$ is rank 3 , it suffices to show $\left(^{*}\right)$ of 2.7. But if $(\alpha, \gamma, \beta)$ is a triangle in $\Omega$, set $X=\langle\alpha, \gamma, \beta\rangle$. Then by $6.3, X / O_{p}(X) \cong S L_{2}(q)$ with $\alpha^{\perp} \cap X=\alpha^{o_{p}(X)}$. So 3.3 yields (*).

Following the notation of D. Higman let $k=\left|D_{\alpha}\right|, l=\left|A_{\alpha}\right|, \lambda=$ $\left|D_{\alpha} \cap D_{\beta}\right|$ for $\beta \in D_{\alpha}$, and $\mu=\left|D_{\alpha} \cap D_{r}\right|$ for $\gamma \in A_{\alpha}$. Let $m=\left|\beta^{M}\right|$. [10] implies:

LEMma 6.7. $l=k(k-\lambda-1) / \mu$ and either

(1) $k=l$ and $\mu=(\lambda+1) / 2=k / 2$ or

(2) $d^{2}=(\lambda-\mu)^{2}+4(k-\mu)$ is a square and $d$ divides $2 k+(\lambda-$ $\mu)(k+l)$.

LEMMA 6.8. $O_{\infty}(L)=Z(L)$. 
Proof. Assume not. Then there exists $x \in O_{\infty}(L)=L \cap M$ with $B^{x} \neq B$. By $6.5, \beta^{x} \neq \beta$, so $\beta^{x} \in\left(\alpha^{*} \beta\right) \cap D_{r}=\{\beta\}$, a contradiction.

LEMma 6.9. $\alpha^{*} \gamma=\langle\alpha, \gamma\rangle \cap \Omega$ has order $q+1$. If $H / M \cong U_{3}(q)$ then $m=q^{2}$.

Proof. Assume $n \geqq 4$. Then a hyperbolic line $\beta \delta$ in $\mathscr{B}(\Gamma)$ is as claimed. But $\beta^{*} \delta \subseteq \beta \delta$ while clearly $\langle\beta, \delta\rangle \cap \Omega \subseteq \beta^{*} \delta$. Next assume $n=2$. Then by $6.3, D_{\alpha} \cap D_{\gamma}=\langle\beta, \delta\rangle \cap \Omega$ for $\beta, \delta \in D_{\alpha} \cap D_{\gamma}$, and $D_{\beta} \cap$ $D_{\delta}=\langle\alpha, \gamma\rangle \cap \Omega$, so $\alpha^{*} \gamma$ is as claimed. Finally assume $H / M \cong U_{3}(q)$. Let $Z=Z\left(\left\langle\alpha^{\perp}\right\rangle\right)$. $\quad Z$ acts semiregularly on $\alpha^{*} \gamma-\{\alpha\}$. So if $\left|\alpha^{*} \gamma\right|=$ $q+1$ then $|Z|=q$. If $\left|\alpha^{*} \gamma\right| \neq q+1$ then $\alpha^{*} \gamma=D_{\beta} \cap D_{\delta}$, for $\beta, \delta \in$ $D_{\alpha} \cap D_{r}$. So $\left|\alpha^{*} \gamma\right|=q^{3}$ and $N_{G}\left(\alpha^{*} \gamma\right)^{\alpha^{*} \gamma}$ acts as a subgroup of Aut $\left(U_{3}(q)\right)$. But by 3.4, $Z$ is elementary abelian, while an elementary subgroup of Aut $\left(U_{3}(q)\right)$ acting semiregularly on $q^{3}$ letters has order at most $q$. Further $\left|\alpha^{*} \gamma\right|-1=\left|N_{M\langle\alpha\rangle}\left(\alpha^{*} \gamma\right)\right|=\left|C_{M\langle\alpha\rangle}(L)\right|=|Z|=q$ by 3.4. So $\left|\alpha^{*} \gamma\right|=q+1$.

Finally $\mu=|\Gamma|=q^{3}+1, \lambda=m-1$, and $k=\mu m$ by 6.3 and 6.8 . Thus by $6.7, q^{3} m^{2}=l$, while by $6.6, l=\left|\left\langle\alpha^{\perp}\right\rangle: N_{\langle\alpha \perp\rangle}(\gamma)\right|=|M\langle\alpha\rangle|=$ $q m^{3}$ by 3.4. Thus $m=q^{2}$.

LEMMA 6.10. If $H / M \cong L_{2}(q)$ then $m=q$ or $q^{2}$. If $H / M \cong S p_{n}(q)$ or $U_{n}(q), n \geqq 3$, then $m=q$ or $q^{2}$ respectively.

Proof. Assume $H / M \cong L_{2}(q)$. Then $\mu=q+1, k=\mu m$ and $\lambda=$ $m-1$. So by $6.7, l=m^{2} q$ and $\mu+\lambda=m+q$ divides $2 k+(\lambda-$ $\mu)(k+l) \equiv-2\left(q^{2}-1\right) q \bmod (m+q)$. By 3.3 , an element of order $q-1$ in $L$ acts semiregularly on $([A, M] / Z)^{\sharp}$ of order $m-1$, so $q-$ 1 divides $m-1$. Thus $q$ divides $m=q^{r+1}$. So $q^{r}+1$ divides $2\left(q^{2}-1\right)$ and therefore $r \leqq 1$. That is $m=q$ or $q^{2}$.

So with 6.9 we can assume $n \geqq 4$. Therefore, singular lines in $L$ have order $q$ or $q^{2}$, respectively. Thus as $\alpha^{*} \beta=\{\alpha\} \cup \beta^{M}$ these lines are also lines in $G$.

\section{LeMma 6.11. $H / M \cong U_{n}(q)$ and $m=q^{2}$.}

Proof. If not $\mu=\lambda+2$, so $\mathscr{B}(\Omega)$ is a symmetric block design. Further all lines have order $q+1$. Thus a result of Dembowski and Wager [8] implies $\mathscr{B}(\Omega)$ is $(n+1)$-dimensional projective space over $G F(q)$. As $G$ is generated by the set of elations of $\mathscr{B}(\Omega)$ commuting with the symplectic polarity $\alpha \leftrightarrow \alpha^{\perp}, G \cong S p_{n+2}(q)$.

The case $n=2$ must be treated differently since in this case the existence of $D$-subgroups isomorphic to $U_{3}(q)$ are not assured. The following lemma treats this special case. 
LEMMA 6.12 . $n \geqq 3$.

Proof. Assume $n=2$. Let $\beta, \delta \in \Gamma$, and set $X=L_{\beta \sigma}$. We first determine the fixed point sets of elements of $L$.

If $x \in\langle\beta\rangle^{\sharp}$ then $F(x)=\beta^{\prime}$. If $x \in X-Z(L)$, then $F(x)=\{\beta, \delta\} \cup$ $\alpha^{*} \gamma$. For if $\sigma \in F(x)$ is not as claimed, then by 3.3, $\sigma \in A_{\alpha}$. $x$ normalizes $\langle\delta, \alpha\rangle \cong S L_{2}(q)$ and centralizes $\alpha$, so $x$ centralizes $\sigma$. Thus a similar argument on $\langle\sigma, \beta\rangle$ and $\langle\sigma, \delta\rangle$ shows $\sigma \in D_{\beta} \cap D_{o}=\alpha^{*} \gamma$. If $\langle x\rangle=$ $Z(L)$ then $F(x)=\Gamma \cup\left(\alpha^{*} \gamma\right)$. For arguing as above $F(x)=C_{\Omega}(x)$, and minimality of $G$ implies $\left\langle C_{\Omega}(x)\right\rangle / Z\left(\left\langle C_{\Omega}(x)\right\rangle\right) \cong L_{2}(q) \times L_{2}(q)$; that is $C_{\Omega}(x)=$ $\Gamma \cup\left(\alpha^{*} \gamma\right)$. Finally let $x \in L$ act fixed point free on $\Gamma$. As above $F(x)=C_{\Omega}(x)$ and as $D_{\alpha} \cap C_{\Omega}(x)$ is empty, $\left\langle C_{\Omega}(x)\right\rangle=Y_{F(x)} \cong S L_{2}(q)$ or $U_{3}(q)$. And if $Y \cong U_{3}(q)$ then $Y$ is doubly transitive so $x \in\left\langle D_{\alpha} \cap D_{\sigma}\right\rangle$ for $\sigma \in F(x)-\{\alpha\}$. Thus $x$ is in $q^{2}$ distinct conjugate of $L$ in $H$. However, with $3.3, C_{M}(x)=\langle\alpha\rangle$, so there are $m^{2} q(q-1) / 2$ conjugates of $\langle x\rangle$ in $H$. On the other hand there are $m^{2}$ conjugates of $L$, each containing $q(q-1) / 2$ conjugates of $\langle x\rangle$, so $\langle x\rangle$ is in a unique conjugate of $L$. So $F(x)=\alpha^{*} \gamma$.

Let $\bar{G}=U_{4}(q)$, let $\bar{D}$ be the class of subgroups generated by transvections in $\bar{G}$, let $\bar{\alpha}$ consists of the members of $\bar{D}$ whose center is a given singular point of the associated projective space, and let $\bar{\Omega}=$ $\bar{\alpha}^{\bar{G}}$. Let $\bar{\gamma} \in A_{\bar{\alpha}}$ and $\bar{L}=\left\langle D_{\bar{\alpha}} \cap D_{\bar{\gamma}}\right\rangle$. The discussion above implies $\bar{L}^{\bar{\mu}}$ is permutation isomorphic to $L^{\Omega}$.

Lemma 6.3 implies that every $\sigma$ in $\Omega-\left(\alpha^{*} \beta\right)$ appears in a unique $D_{\beta_{1}}, \beta_{1} \in \alpha^{*} \beta$. Set $K=L_{\beta}$, and let $t \in L$ have cycle $(\beta, \delta)$. Let $\sum_{i=0}^{q+2} \beta_{i}^{K}$ be a partition of $\alpha^{*} \beta$ with $\beta_{0}=\alpha$ and $\beta_{1}=\beta$. Set $\Lambda_{i}=\left(\beta_{i}-\left(\alpha^{*} \beta\right)\right) \cup$ $\left\{\beta_{i}\right\}$, and $\Lambda=U \Lambda_{i}$. Then $L$ maps the edge set of $\mathscr{D}(\Lambda)$ onto the edge set of $\mathscr{D}(\Omega)$, except for edges in $\mathscr{D}\left(\alpha^{*} \beta\right)$.

Let $T$ be permutation isomorphism of $L$ and $\bar{L}$, and let $\bar{\beta}=\beta T$. Let $\bar{\beta}_{i}^{K T}$ be orbits of $K T$ on $\bar{\alpha}^{*} \bar{\beta}$ and define $\bar{A}$ as above with respect to these $\bar{\beta}_{i}$. There exists an isomorphism $S$ of $\mathscr{D}(\Lambda)$ and $\mathscr{D}(\bar{\Lambda})$ such that $S$ restricted to $\mathscr{D}\left(\Lambda_{i}\right)$ commutes with $T$ restricted to $N_{L}\left(\Lambda_{i}\right)$ and $N_{\bar{L}}(\sigma S)=$ $\left(N_{L}(\sigma)\right) T$ for $\sigma \in \Lambda$. For $\sigma \in \Lambda_{i}$ there exists $\bar{\sigma} \in \bar{\Lambda}_{i}$ with $N_{\bar{L}}(\bar{\sigma})=\left(N_{L}(\bar{\sigma})\right) T$ from the discussion above, so $S$ can be defined in the obvious manner. So we can apply 2.6 to show $\mathscr{D}(\Omega) \cong \mathscr{D}(\bar{\Omega})$ and thus $G \cong \bar{G}$, if we show condition (ii) of 2.6 is satisfied.

Clearly (ii) holds on $\Lambda_{0}$. Suppose $\sigma, \sigma^{x} \in \Lambda_{1}, x \in L$. Claim $\sigma^{x}=\sigma^{y}$ for $y \in K$. As $L=K \cup K t K$ we can assume $x=t$. Thus $\sigma^{x} \in D_{\beta} \cap D_{\hat{o}}=$ $\alpha^{*} \gamma$, so $\sigma=\sigma^{t}$ is fixed by $t$. But $K=N_{L}\left(\Lambda_{1}\right)$, so (ii) holds here. Suppose $\sigma, \sigma^{x} \in \Lambda_{i}, i \geqq 2$. We consider the case $\left|\sigma^{L}\right|=q^{2}-1$; the case $\left|\sigma^{L}\right|=q\left(q^{2}-1\right)$ is analogous. Now $\langle\beta\rangle=N_{L}\left(\Lambda_{i}\right)$ and $q^{2}=\left|\Lambda_{i} \cap \bigcup_{\alpha^{*} \gamma} D_{\omega}\right|$ in $q$ orbits of length $q$ under $\langle\beta\rangle$. These are the points in orbits of length $q^{2}-1$ under $L$. Let $\theta$ be the set of edges $\left(\beta_{i}^{y}, \omega\right)$ with $y \in L$ 
and $\left|\omega^{L}\right|=q^{2}-1$. Let $N$ be the number of orbits of $L$ on $\theta$. Then $q\left(q^{2}-1\right) N=\left|\left(\beta_{i}, \sigma\right)^{L}\right| N=|\theta|=\left|\beta_{i}^{L}\right| q^{2}=\left(q^{2}-1\right) q^{2}$, so $N=q$. Thus $\left(\beta_{i}, \sigma^{x}\right)=\left(\beta_{i}, \omega^{y}\right)$ for some $\omega \in \Lambda_{i}, y \in\langle\beta\rangle$. That is condition (ii) holds on $\Lambda_{i}$.

This completes the proof of 6.12 .

A unitary $(\alpha, \beta, \gamma)$ in $\Omega$ is a triple with $\beta \in A_{\alpha}$ and

$$
\gamma \in \bigcap_{\delta \in \alpha^{*} \beta} A_{\delta} \text {. }
$$

LEMMA 6.13. If $(\alpha, \beta, \gamma)$ is a unitary triple then $\langle\alpha, \beta, \gamma\rangle \mid Z(\langle\alpha$, $\beta, \gamma\rangle) \cong U_{3}(q)$.

Proof. We can choose a unitary triple $\left(\beta_{1}, \beta_{2}, \beta_{3}\right)$ in $H$. Set $X=$ $\left\langle\beta_{1}, \beta_{2}, \beta_{3}\right\rangle$. As $H / M \cong U_{n}(q), X / Z(X) \cong U_{3}(q)$. If $n=3$ we can count the number of unitary triples and the number of such triples centralizing some $\alpha \in \Omega$. These two numbers are equal. So assume $n \geqq 4$, and let $\left(\sigma_{1}, \sigma_{2}, \sigma_{3}\right)$ be a unitary triple. Choose $\beta \in D_{\sigma_{1}} \cap D_{\sigma_{2}}$. If $\sigma_{3} \in$ $D_{\sigma}$ set $\beta=\alpha$. If not let $\alpha^{*} \beta$ be a singular line in $D_{\sigma_{1}} \cap D_{\sigma_{2}} \cdot$ By 6.3, we can assume $\alpha \in D_{\sigma_{3}}$. Thus as above we are through.

Let $(\alpha, \gamma, \delta)$ be a unitary triple in $D_{\beta}$. Set $J=\left\langle D_{\hat{o}} \cap \Gamma\right\rangle$.

LEMMA 6.14. $J / Z(J) \cong U_{n-1}(q)$.

Proof. If $n=3,\langle\alpha, \gamma, \delta\rangle=D_{\beta} \cap D_{\sigma}$ for suitable $\sigma \in A_{\beta}$ and $J=$ $\left\langle\beta^{*} \sigma\right\rangle$. If $n=4, J$ has width one and a counting argument shows $|J \cap \Omega|=q^{3}+1$. Thus by minimality of $G, J / Z(J) \cong U_{3}(q)$. Finally if $n>4$, then arguing as in $6.3, J$ is transitive on $J \cap D$ and $\left\langle D_{\beta} \cap\right.$ $J\rangle / O_{\infty}\left(\left\langle D_{\beta}\right\rangle\right) \cong U_{n-3}(q)$, so minimality of $G$ implies the desired result.

Lemma 6.15. Let $\theta=\Gamma \cup \delta^{L}$ and $K=\langle\theta\rangle$. Then $K \cong S U_{n+1}(q)$ and $\Omega=\theta \cup \alpha^{K}$.

Proof. Claim $\theta^{\theta}=\theta$. Clearly $L$ normalizes $\theta$, so it suffices to show $\delta$ normalizes $\theta$. Let $\sigma \in \Gamma \cap A_{\grave{\delta}}$. Then $\langle\sigma, \delta\rangle \cong S L_{2}(q)$, so $\sigma^{\delta}=$ $\delta^{\sigma} \subseteq \theta$. Thus $\Gamma^{\delta} \subseteq \theta$. Using the fact that 6.15 is true in $U_{n+1}(q)$, one can check that

$$
L=J\left(\bigcup_{\ell}\left\langle\sigma_{1}^{*} \sigma_{2}\right\rangle\right)
$$

where $\mathscr{L}$ is the set of lines in $L-J$. Thus it suffices to show $X \cap$ $\Omega \subseteq \theta$ when $X=\left\langle\sigma_{1}, \sigma_{2}, \delta\right\rangle$. But if $\left(\sigma_{1}, \sigma_{2}, \delta\right)$ is unitary, 6.13 implies $X \cap \Omega=\sigma_{1}{ }^{*} \sigma_{2} \cup \delta^{\left\langle\sigma_{1}{ }^{*} \sigma_{2}\right\rangle} \cong \theta$ and if $\left(\sigma_{1}, \delta, \sigma_{2}\right)$ is a triangle then $X / O_{p}(X) \cong$ $S L_{2}(q)$ and 3.3 yields the same equality.

So $\theta^{\theta}=\theta . \quad \alpha \notin \theta$, so $K \neq G . \quad Y=\left\langle D_{\beta} \cap \theta\right\rangle=\left\langle D_{\beta} \cap \Gamma\right.$, $\left.\delta\right\rangle$, so 
$Y / O_{\infty}(Y) \cong U_{n-1}(q) . \quad[L, \alpha]=1$ and $\delta \in A_{\alpha}$, so $\Gamma=D_{\alpha} \cap \theta$. Arguing as above $\theta \cup \alpha^{K}$ is self normalizing, so $\Omega=\theta \cup \alpha^{K}$.

Let $Z=Z(K) . \quad Z$ fixes $\theta$ pointwise and $K \leqq C_{G}(Z)$ is transitive on $\Omega-\theta$, so $Z$ does not fix $\alpha$. $\left|S U_{n+1}(q)\right| /\left|S U_{n}(q)\right|=\left|\alpha^{K}\right|=\left|K: N_{K}(\alpha)\right|$ and $L Z / Z \cong S U_{n}(q)$, so $|Z|=(n+1, q)$. Considering the covering group of $U_{n+1}(q)$ we get $K \cong S U_{n+1}(q)$.

Put $K$ and $D_{\delta}$ in the roles of $H$ and $A$ in 2.6. Then 6.15 and 5.4 together with 2.6 imply $G \cong U_{n+2}(q)$.

This completes the proof of the main theorem.

\section{REFERENCES}

1. M. Aschbacher, Doubly transitive groups in which the stabilizer of two points is abelian, J. Algebra. 18 (1971), 114-136.

2. - On doubly transitive permutation groups of degree $n \equiv 2 \bmod 4$, Illinois J. Math., 16 (1972), 276-279.

3. - Finite groups generated by odd transpositions, (to appear).

4. M. Aschbacher and M. Hall, Jr., Groups generated by a class of elements of order 3, (to appear, J. Algebra).

5. H. Bender, Endliche zweifach transitive Permutationsgruppen, deren Involutionen keine Fixpunkte haben, Math. Zeitschr., 104 (1968), 175-204.

6. - Transitive Gruppen gerader Ordnung in denen jede Involution genau eineu Punkt feslast, J. Algebra, 17 (1971), 527-554.

7. R. Brauer and C. Nesbitt, On the modular characters of groups, Ann. of Math., 42 (1941), 556-590.

8 P. Dembowski and A. Wagner, Some characterizations of finite projective space, Arch. Math., 11 (1960), 465-469.

9. G. Glauberman, Central elements in core-free groups, J. Algebra, 4 (1966), 403-420. 10. D. Higman, Finite permutation groups of rank 3, Math. Zeitscher., 86 (1964), $145-156$.

11. W. Kantor and G. Seitz, Finite groups having a split BN-pair of rank 1, (to appear). 12. - Some results on 2-transitive groups, Inventiones Math., 13 (1971), 125-142. 13. J. Walter, The characterization of groups with abelian Sylow 2-subgroups, Ann. Math., 89 (1969), 405-514.

Received February 25, 1972 and in revised form February 26, 1973.

California Institute of Technology 


\section{PACIFIC JOURNAL OF MATHEMATICS}

\section{EDITORS}

D. Gilbarg and J. Milgram

Stanford University

Stanford, California 94305

R. A. Beaumont

University of Washington

Seattle, Washington 98105
J. DUGUNDJI* Department of Mathematics

University of Southern California Los Angeles, California 90007

RICHARD ARENS

University of California

Los Angeles, California 90024

\section{ASSOCIATE EDITORS}

E. F. BeCKenbach

B. H. NeUMaNN

F. WOLF

K. Yoshida

\section{SUPPORTING INSTITUTIONS}

UNIVERSITY OF BRITISH COLUMBIA

UNIVERSITY OF SOUTHERN CALIFORNIA

CALIFORNIA INSTITUTE OF TECHNOLOGY

UNIVERSITY OF CALIFORNIA

MONTANA STATE UNIVERSITY

STANFORD UNIVERSITY

UNIVERSITY OF TOKYO

UNIVERSITY OF NEVADA

UNIVERSITY OF UTAH

NEW MEXICO STATE UNIVERSITY

WASHINGTON STATE UNIVERSITY

OREGON STATE UNIVERSITY

UNIVERSITY OF OREGON

OSAKA UNIVERSITY

UNIVERSITY OF WASHINGTON

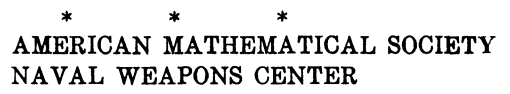

* C. DePrima will replace J. Dugundji until August 1974. 


\section{Pacific Journal of Mathematics}

\section{Vol. 47, No. $1 \quad$ January, 1973}

K. Adachi, Masuo Suzuki and M. Yoshida, Continuation of holomorphic

mappings, with values in a complex Lie group ....................

Michael Aschbacher, A characterization of the unitary and symplectic groups

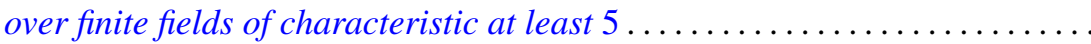

Larry Eugene Bobisud and James Calvert, Energy bounds and virial theorems for abstract wave equations....................................

Christer Borell, A note on an inequality for rearrangements ................

Peter Southcott Bullen and S. N. Mukhopadhyay, Peano derivatives and general

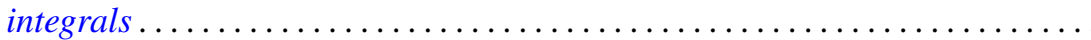

Wendell Dan Curtis, Yu-Lee Lee and Forrest Miller, A class of infinite dimensional subgroups of $\operatorname{Diff}^{r}(X)$ which are Banach Lie groups .........

Paul C. Eklof, The structure of ultraproducts of abelian groups ...............

William Alan Feldman, Axioms of countability and the algebra $C(X) \ldots \ldots \ldots$

Jack Tilden Goodykoontz, Jr., Aposyndetic properties of hyperspaces...........

George Grätzer and J. Płonka, On the number of polynomials of an idempotent algebra. II ...........................................

Alan Trinler Huckleberry, The weak envelope of holomorphy for algebras of

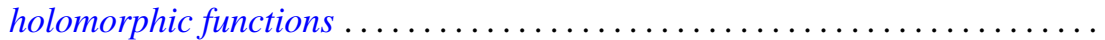

John Joseph Hutchinson and Julius Martin Zelmanowitz, Subdirect sum decompositions of endomorphism rings . . . . . . . . . . . . . . . .

Gary Douglas Jones, An asymptotic property of solutions of

$y^{\prime \prime \prime}+p y^{\prime}+q y=0$.

Howard E. Lacey, On the classification of Lindenstrauss spaces .

Charles Dwight Lahr, Approximate identities for convolution measure algebras.

George William Luna, Subdifferentials of convex functions on Banach

spaces.

Nelson Groh Markley, Locally circular minimal sets. .

Robert Wilmer Miller, Endomorphism rings of finitely generated projective modules

Donald Steven Passman, On the semisimplicity of group rings of linear

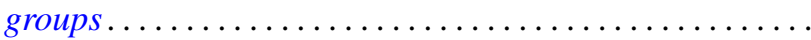

Bennie Jake Pearson, Dendritic compactifications of certain dendritic spaces.

Ryōtarō Satō, Abel-ergodic theorems for subsequences ...... .

Henry S. Sharp, Jr., Locally complete graphs. . .

Harris Samuel Shultz, A very weak topology for the Mikusinski field of

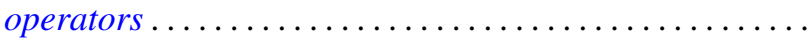

Elena Stroescu, Isometric dilations of contractions on Banach spaces ...

Charles W. Trigg, Versum sequences in the binary system ... . .

William L. Voxman, On the countable union of cellular decompositions of n-manifolds 